\title{
Improving Alarm Management Efficacy through Predictive Modeling and Trending
}

Edward J. Ryan IV

EJRyan89@gmail.com

\section{Recommended Citation}

Ryan, Edward J. IV, "Improving Alarm Management Efficacy through Predictive Modeling and Trending" (2014). Master's Theses. 566. https://opencommons.uconn.edu/gs_theses/566

This work is brought to you for free and open access by the University of Connecticut Graduate School at OpenCommons@UConn. It has been accepted for inclusion in Master's Theses by an authorized administrator of OpenCommons@UConn. For more information, please contact opencommons@uconn.edu. 


\section{Improving Alarm Management Efficacy through Predictive Modeling and Trending}

Edward Joseph Ryan IV

B.S., University of Connecticut, 2012

A Thesis

Submitted in Partial Fulfillment of the

Requirements for the Degree of

Master of Science at the

University of Connecticut

2014 


\title{
APPROVAL PAGE
}

Masters of Science Thesis

\section{Improving Alarm Management Efficacy through Predictive Modeling and Trending}

\author{
Presented by
}

Edward Joseph Ryan IV, B.S.

Major Advisor:

Dr. John D. Enderle

Associate Advisor:

Dr. Quing Zhu

Associate Advisor:

Frank Painter

University of Connecticut 


\section{Acknowledgements}

This thesis would not have been possible without the time, advice, and patience of members of staff at Hartford Hospital. The Biomedical Engineering Department, as a whole, was very supportive throughout the thesis process, whether it was answering technical questions regarding the patient monitoring system, clarifying hospital policies, or providing moral support and constructive feedback. In particular, Ray Renouf was an invaluable resource, providing both technical expertise regarding all aspects of the project and a great deal of advice regarding the thesis process as a clinical engineer. Amato DeRosa facilitated several efforts to increase awareness in the hospital regarding alarm fatigue, and eventually helped with the formation of the hospital's alarm management committee. Sean Frenette and Ruth Zafian provided technical and clinical advice, respectively, and together have helped to make the outcomes of this thesis into a foundation for the work of the alarm management committee. Also, thank you to Kevin Walsh, Michael Ballintyn, and Yvonne Nanakos for being patient with me and providing constructive feedback at numerous points throughout the project.

There were several individuals outside of the hospital that were also crucial to completing this thesis. Richard Crane, of Excel Medical Electronics, was very forthcoming with information regarding the data manipulation mechanics within BedMasterEX. Frank Painter provided a background for numerous aspects of clinical engineering, and was helpful in providing networking opportunities with experts in the alarm management arena. Dr. John Enderle and Dr. Quing Zhu were supportive as advisors, ensuring that all requirements were met by their appropriate deadlines.

Finally, thank you to my classmates, friends, and family for their patience and words of encouragement throughout this entire thesis process. 


\section{Table of Contents}

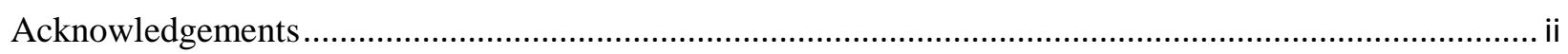

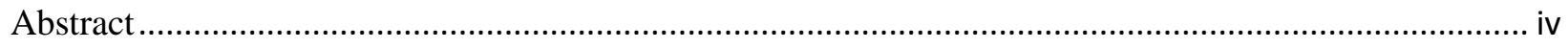

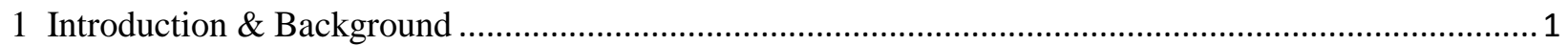

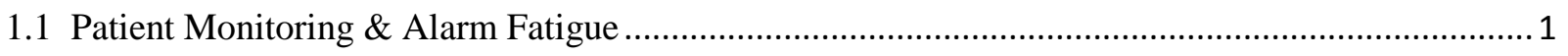

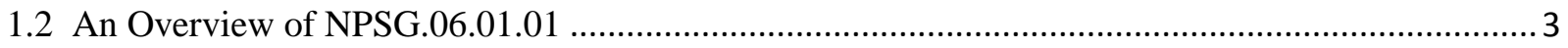

1.3 General Electric (GE) Healthcare's Patient Monitoring Technology .............................................. 7

1.4 Clinical Alarm Monitoring Challenges ......................................................................................

1.5 Analyzing Physiological Alarms at Hartford Hospital ........................................................... 10

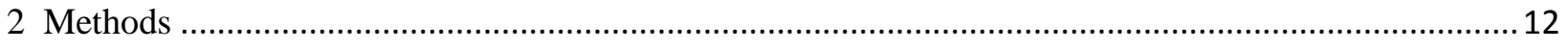

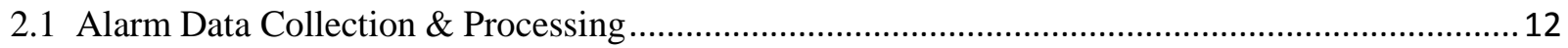

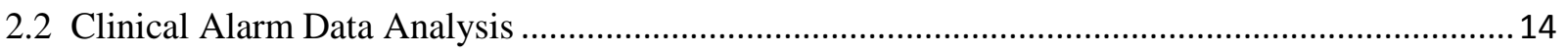

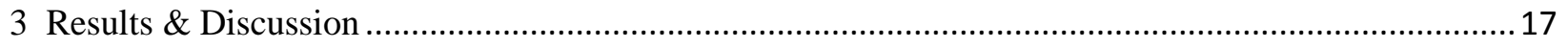

3.1 Physiological Alarm Frequency Distributions ….................................................................... 17

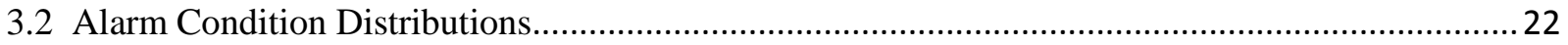

3.3 Comparing Coefficients of Determination $\left(\mathrm{R}^{2}\right)$ Across Acuity Levels........................................26

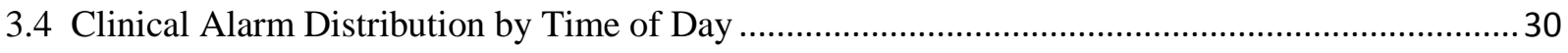

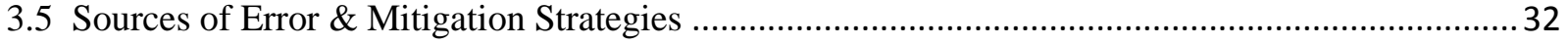

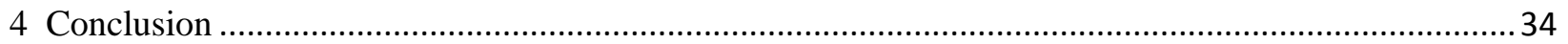

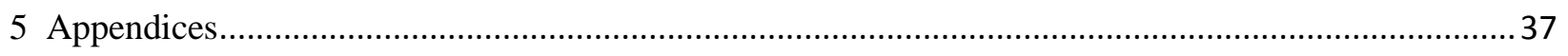

Appendix A - Clinical Definitions of Select Cardiac Arrhythmia Alarms............................................37

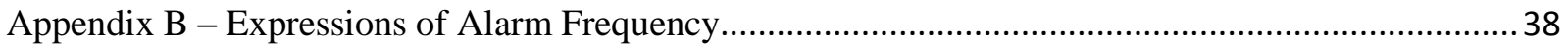

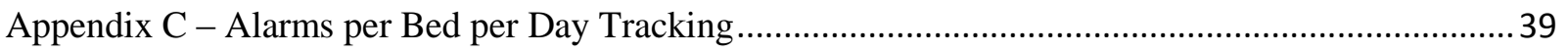

Appendix D - Detailed Acuity Level Alarm Frequency Distributions............................................... 40

Appendix E - Detailed Alarm Condition Distributions ........................................................................ 43

Appendix F - Coefficients of Determination $\left(\mathrm{R}^{2}\right)$ for Select Distributions ........................................ 47

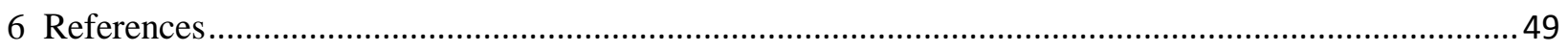




\section{Abstract}

Alarm fatigue, the progressive desensitization of clinical staff to audible alarms in their environment, has been re-established as a National Patient Safety Goal by The Joint Commission as of January 1, 2014. In order to manage the number of alarms experienced by hospital employees, facilities are charged with finding a way to monitor their existing alarm load and then develop methods and policies by which to reduce or mitigate the threat of alarm fatigue. This study used an archive of 9.76 million patient monitoring alarms collected over an eight-month period in order to develop a process by which patient monitoring alarms could be analyzed with high specificity across multiple units of differing specialties and acuity levels.

Trends in the distributions of patient monitoring alarm data were identified, with the greatest potential contributors to alarm fatigue being confirmed as the medium and low priority alarms, comprising greater than $70 \%$ of all patient monitoring alarms observed, and conditionbased distributions showed strong correlation to average distributions across patient care units of differing specialties and acuity levels. These distributions demonstrated the expected exponential decay in frequency of alarms as the condition shifted farther away from the physiologically acceptable range, with one exception. A trend of spikes in high heart rate alarms at heart rate values that were multiples of 10 was observed, and at multiples of five for low heart rate alarms, suggesting that heart rate limits should be considered differently than those for other vital signs. The time of day in which alarms initiated was also analyzed, showing a direct relation between periods of time where patients are interacted with by clinical and/or support staff, with a physiological sensitivity to these interactions increasing as the acuity level of the unit increased. 


\section{Introduction \& Background}

A general understanding of the role of patient monitoring in an acute care environment is necessary when discussing the dangers posed by alarm fatigue with this system. This, in turn, will help to explain the need for patient monitoring alarm management, why The Joint Commission made alarm system safety a National Patient Safety Goal, and provide context for the study performed at Hartford Hospital.

\subsection{Patient Monitoring \& Alarm Fatigue}

A cornerstone of many inpatient care units, especially at higher acuity levels, is the patient monitoring system. The clinical staff is able to observe a patient's condition, via his or her vital signs, without having to be physically present in the patient's room. Adverse changes are identified by the patient monitor, and the staff is informed by one or more corresponding alarms. These alarms can be divided into two major categories: physiological alarms, which directly detail a patient's condition, and technical alarms, which are in reference to the patient monitor or its accessories.

The general mentality in the hospital environment is that "more information is better," and this is supported by how the alarm algorithms are structured. Patient monitoring alarms are designed to maintain high sensitivity, a preventative measure to minimize the risk of false negative alarms, or missed true events. This is done at the expense of specificity, which results in frequent false positive alarms. A study conducted by Chambrin et al. quantified this situation, finding that the observed patient monitoring systems exhibited a sensitivity of $97 \%$ (3\% of alarmed events are missed), and a specificity of $58 \%$ (42\% of positive alarms are false) [1]. The result is a large quantity of alarms that do not require any clinical intervention, which can lead to 
alarm fatigue and a "cry wolf" effect [2]. Alarm fatigue, for the context of this study, refers to the progressive desensitization of hospital staff to audible alarms in their environment. This can lead to delayed response times, missed alarms, or impaired decision-making when responding to an alarm [3,4]. The "cry wolf" effect is similar in the complications it creates, but relates specifically the development of caregiver apathy in relation to the alarms. This apathy can lead to both delayed responses and the disabling of alarm systems [2].

Though alarm fatigue has been included in research literature for many years [3], national attention to the issue has risen with the publicizing of patient deaths directly related to failure to respond to clinical alarms. One of the first of these publicized incidents was the death of Madeline Wagner at the University of Massachusetts Memorial Medical Center (Worcester, MA) in January 2007, where the patient's monitor sounded a low battery alarm, to which the clinical staff did not respond. She suffered cardiac arrest, and with no power to the monitor to generate an alarm, the staff remained unaware and the patient expired [5]. A similar incident occurred at the same facility in August 2010, where a patient suffered serious brain damage (and eventually death) as the result of failure to respond to high heart rate and low oxygen saturation alarms [5]. One of the most highly publicized issues was an incident at Massachusetts General Hospital (Boston, MA) in January 2010. In this case, a patient's bedside monitor had certain high priority alarms turned off the previous night, so that when his heart rate fell and eventually stopped, the clinical staff was not notified [6].

These events represent a small sample of documented patient deaths associated with alarm fatigue. The Food \& Drug Administration's (FDA) Manufacturer and User Facility Device Experience (MAUDE) database contains 566 reports of patient deaths directly associated with monitoring device alarms from 2005 to 2008 [3]. This number is likely far lower than the actual 
number of patient deaths, as reporting to this system is voluntary, and experts consider these incidents to be underreported [4].

Patient death is not the only concern associated with alarm fatigue. A study from Taenzer et al. discussed the connection of alarm fatigue to the total number of rescue events [7]. These do not necessarily result to patient loss, but do account for increased care costs due to patients being transferred to higher acuity levels, increased length of stay, or other complications associated with the patient's deteriorated condition.

The need for methods by which to mitigate the threat of alarm fatigue is growing as more and more hospitals, agencies, and manufacturers collect information about the alarms in the care environment. The Emergency Care Research Institute (ECRI) has labeled "Alarm Hazards" as either its first or second "Top 10 Health Technology Hazard" from 2010 to 2013, with reports on the issue appearing since 2007 [4,8]. The Joint Commission, the primary accrediting agency for most healthcare organizations, issued a "Sentinel Event Alert" in April 2013 calling for hospitals to address alarm safety in their facilities [4]. In addition, the National Patient Safety Goal (NPSG) NPSG.06.01.01 was reinstated, with Element of Performance deadlines beginning in 2014, making alarm management a required priority for hospitals nationwide [9].

\subsection{An Overview of NPSG.06.01.01}

By creating NPSG.06.01.01, hospitals are now required to care about alarm fatigue within their institutions. National Patient Safety Goals are scorable criteria for hospital accreditation, and failure to meet these requirements, itemized as Elements of Performance, can result in mandated corrective actions, fines, or in extreme cases, loss of accreditation. Noncompliance can also impact interactions with other organization, such as the Department of Public Health (DPH) for the state in which the hospital resides, as they often use The Joint 
Commission's requirements and standards as a basis for their own inspection criteria, and the Centers for Medicare \& Medicaid Services (CMS), the organization responsible for reimbursing hospitals for the care provided to the patients they cover. This creates a financial incentive for hospitals to fully comply with NPSG.06.01.01 and all other National Patient Safety Goals.

The National Patient Safety Goal is generalized to be applicable to all clinical alarm systems, though many facilities, including Hartford Hospital, are starting by addressing patient monitoring alarms. This is in part because of the high visibility of the system, as well as the potential for patient death if a critical alarm is missed or the system malfunctions. Patient monitoring systems also have more data centralization than other devices, and as such, allow for easier collection of alarm data.

The first Element of Performance (EP1) of NPSG.06.01.01 requires hospital leadership to establish alarm system safety as a hospital priority [9]. This mandates the identification of a new or existing body to be responsible for determining the efficacy of alarm systems currently in place in the hospital and to ensure that new systems being installed have had their alarm settings and policies analyzed. Hartford Hospital has responded to this by forming an alarm management committee, along with defining the reporting process for other groups in the hospital that validate the committee's recommendations.

Element of Performance 2 (EP2) requires a prioritization of existing alarms and alarm systems in the hospital environment based on, at minimum, the criteria described in the Element of Performance [9]. This results in an alarm inventory document, outlining each of the alarm systems present in the hospital. The focus of this inventory is geared towards those alarms in patient care areas, but is not restricted to this subset. The alarm inventory is meant to include a process for updating it based on internal incident analysis, new publications regarding best 
EP1 - As of July 1, 2014, leaders establish alarm system safety as a hospital priority.
Need to provide evidence of hospital leadership acknowledging the risk of alarm system safety

- Identified as a risk area

- Structural (Category A) scoring

Need to provide evidence of alarm prioritization

- Identified as a risk area

- Structural (Category A) scoring

- Risk to patients if the alarm signal is not attended to or it malfunctions

- Whether specific alarm signals are needed or unnecessarily contribute to alarm noise and alarm fatigue

- Potential for patient harm based on internal incident history

- Published best practices and guidelines

EP3 - As of January 1, 2016, establish policies and procedures for managing the alarms identified in EP 2 above that, at a minimum, address the following:

- Clinically appropriate settings for alarm signals

- When alarm signals can be disabled

- When alarm parameters can be changed

- Who in the organization has the authority to set alarm parameters

- Who in the organization has the authority to change alarm parameters

- Who in the organization has the authority to set alarm parameters to "off"

- Monitoring and responding to alarm signals

- Checking individual alarm signals for accurate settings, proper operations, and detectability

EP4 - As of January 1, 2016, educate staff and licensed independent practitioners about the purpose and proper operation of alarm systems for which they are responsible
Need to formally define policies and procedures for managing alarm systems for various devices and systems

- Identified as a risk area

- Structural (Category A) scoring

- Written documentation of policies required for compliance
Need to demonstrate staff knowledge of manipulating alarm systems which they are allowed to change

- Identified as a risk area

- Category C scoring

Table 1.1: The elements of performance for NPSG.06.01.01 are shown, along with descriptions of the scoring requirements [9]. Note that Category A requirements are scored as either met or not met, while Category C requirements are scored based on the number of instances of noncompliance. 
practices, new and updated technologies, and other factors. This study aims, in part, to assist in prioritizing patient monitoring alarms.

The third Element of Performance (EP3) requires that policies and procedures be formally documented regarding how the alarm systems identified in the alarm inventory are to be managed [9]. Of the requirements here, defining specific criteria for what settings are clinically appropriate has resulted in an effort to examine existing clinical alarm settings and determine if limits, criticality levels, and other setting may be adjusted to a range that reduces the number of false or inactionable alarms. This process requires developing a method by which to catalogue what alarms are occurring, which does not exist for many alarm systems. This study presents a method by which patient monitoring alarm data is able to be captured and analyzed to provide data to support recommendations to develop more clinically appropriate alarm settings.

The final Element of Performance (EP4) addresses staff education regarding the alarm systems for which they are responsible [9]. Though there is a deadline associated with it, the education effort is meant to be ongoing, with individuals being updated as systems are changed, replaced, or removed. This is the only Element of Performance in the National Patient Safety Goal that is not scored simply met or not met. It is instead based on the number of times that the Element of Performance is not met, with the scoring penalty increased if it is consistently not met. This is designed to encourage the education to be ongoing, and ensure that staff is kept updated. This study aims to provide analysis that shows how individual patient monitoring alarms function, thereby helping staff to better understand the impact that specific settings have on the alarm. 


\subsection{General Electric (GE) Healthcare's Patient Monitoring Technology}

As Hartford Hospital uses some form of GE Healthcare's patient monitoring equipment for all acuity levels, it is important to have a basic understanding of how GE Healthcare's patient monitoring system handles its alarms, and how this manifests itself at Hartford Hospital.

Hartford Hospital primarily used, at the time of this study, a combination of Dash 3000/4000/5000 and SOLAR 8000i bedside monitors as well as ApexPro telemetry systems for monitoring patients. These monitors fed information back to Clinical Information Centers (CICs) (V4.0, V5.0, or V5.1), central viewing stations with audiovisual feeds to designated locations for remote patient monitoring. The system used a network architecture based on two parallel networks: the Mission Critical (MC) network, where patient data is transferred, and the Information Exchange (IX) network, which is used for remote access of CICs and other nonmission critical features. All Dash and SOLAR monitors, CICs, and telemetry servers have MC connections, while only CICs and telemetry servers have IX connections.

The physiological alarms for the patient monitoring system fall into one of two categories: parameter alarms and arrhythmia alarms. Parameter alarms are based on pre-defined limits for different vital signs measurements. For all continuously monitoring vital signs (everything except for non-invasive blood pressure), the number compared against the limit is an eight-second moving average from the applicable waveform. If this value reaches or exceeds this limit for five or more seconds, the alarm will sound. Arrhythmia alarms are exclusive to the electrocardiogram (ECG) waveform, and are based on pattern recognition of pre-established arrhythmias on a three-second time delay.

The patient monitors allow for control over two types of alarm settings for physiological alarms: limits and priorities. The alarm limits are those associated with the parameterized alarms, 
determine at what value the alarm will begin to sound. Default limits are stored on the monitor, but may be changed by the clinical staff for once a patient is admitted, returning to the default limits upon patient discharge. The alarm priority dictates the criticality of the alarms. Physiological alarms can be assigned at one of four priorities, each with a different response by the monitor: Crisis, Warning, Advisory and Message (see Table 1.2). Each alarm priority is meant to convey a different level of urgency to the clinical staff. Crisis alarms denote a lifethreatening event, one that requires immediate clinical intervention. Warning alarms denotes a situation that may pose imminent danger to the patient. Potentially unsafe changes in a patient's vital signs trigger advisory alarms, while message alarms are meant to signal that a certain pattern or trend in the patient's vital signs has been identified [11].

At Hartford Hospital, the Medical/Surgical (Med/Surg) units primarily use telemetry for monitoring their patients, while the Stepdown and Intensive Care Units (ICUs) rely on the SOLAR 8000i monitors, with telemetry sometimes used when ambulating a patient. Dash monitors are available on some telemetry units, though are more prevalent in perioperative areas. The Dash and SOLAR monitors collect data on multiple vital signs, including ECG, oxygen saturation, non-invasive blood pressure, and others, provided the proper modules are installed. The ApexPro telemetry monitors are only able to capture ECG, though more recent versions (that were not used in this study) are also able to collect pulse oximetry data.

\begin{tabular}{|c|c|c|}
\hline Alarm Priority & Number of Audible Tones in a Series & Displayed Flashing Text \\
Crisis & Three & Yes \\
Warning & Two & Yes \\
Advisory & One & Yes \\
Message & None & Yes \\
\hline
\end{tabular}

Table 1.2: The audiovisual response of the monitor and central station for alarms of each priority level is shown. Crisis priority alarms are also "latched," meaning they can only be silenced from the bedside, while lower priority alarms can be silenced remotely. 


\subsection{Clinical Alarm Monitoring Challenges}

GE Healthcare's patient monitoring system provides up to 72 hours of patient data disclosure ("full disclosure"), but the availability of this information does not translate well to extended alarm data monitoring, as it is no longer available after the licensed period, or if the patient is discharged. To circumvent this issue, a third-party program, BedMasterEX (V4.1.8) (Excel Medical Electronics, Jupiter, FL), was used to archive patient data beyond the full disclosure period. Using read-only access to the MC network, BedMasterEX collects episodic vital signs, continuous waveform, and alarm data and stores it on a separate server. From here, the client program allows credentialed users access to data for all reporting monitors on the network. The time span of data available from BedMasterEX is dependent on the storage space on the server, as opposed to a time limit set by licensing.

BedMasterEX is capable of generating an alarm history for a set of pre-defined time periods (today, yesterday, this week, last week, this month, last month, or last 100 alarms), which can be exported into Microsoft Excel. Each alarm instance comes with several pieces of information: the date and time the alarm triggered, the patient's name, the patient's identification number, and the alarm message. The alarm message is comprised of the name of the alarm triggered (as it appears on the monitor), the associated numeric condition (for parameter alarms only), and the duration of the alarm. The numeric condition displayed is the value at which the alarm initiated, meaning that an alarm of "HR HI 121" is a high heart rate alarm that, after the five second time delay, was triggered when the average patient's heart rate was 121 . The duration of the alarm denotes how long until the alarm was stopped, or the alarm condition was no longer met (i.e. the vital sign returned to a physiologically acceptable range). 


\subsection{Analyzing Physiological Alarms at Hartford Hospital}

This study was designed to develop a comprehensive set of baseline data for patient monitoring alarms at Hartford Hospital from which future alarm reduction efforts could be compared. Particularly in higher acuity areas, patient stays can last multiple weeks, so to obtain a profile of what alarms would come from an average patient in that unit, data collection needed to occur over an extended period of time and from multiple sources. To accommodate these conditions, a generalized set of requirements was created for inclusion of units in the data collection process. To be eligible for inclusion in the study, units needed to be currently using SOLAR 8000i bedside monitors, Dash 3000/4000/5000 bedside monitors, or ApexPro telemetry for monitoring their patients. The alarm data from these monitors needed to be available for collection from BedMasterEX, and the overall monitoring uptime on the unit had to be greater than $50 \%$. The last criterion was a non-issue for ICU and Stepdown units, as continuous monitoring is standard for all patients. It was instead meant to filter out Med/Surg units that did not regularly use telemetry.

In addition to selecting multiple units at each acuity level that regularly incorporated continuous monitoring as part of their plans of care, the length of the data collection period had to be sufficient in duration to mitigate the spikes in the frequency of some alarms as the result of an unstable patient, or from increases in patients with admitted conditions that are associated with a particular part of the calendar year. To obtain this less-biased data set, the data collection process was extended to last over an eight month period: March 1, 2013 through October 31, 2013. This time period covered multiple seasons, and demonstrated adequate patient turnover to account for small spikes in the frequency of specific alarms. 
The intent of this data collection was to look at the prevalence of alarms that were considered to have the greatest likelihood of generating clinically insignificant alarms. Other studies focused on parameter alarms for commonly monitored vital signs, such as heart rate, peripheral oxygen saturation $\left(\mathrm{SpO}_{2}\right)$, and non-invasive blood pressure, along with arrhythmia alarms of similar priority level [7,11]. These alarms were included in the scope of the study, along with respiratory rate, which, in initial observations of alarm histories, appeared frequently enough to merit inclusion. The end result was a subset of 18 alarms that had their specific frequencies tracked in addition to the total number of alarms for the unit (see Table 1.3). Appendix A contains the clinical definitions of the in-scope arrhythmia alarms for adult patients, as they are recognized by GE Healthcare [12].

\begin{tabular}{|l|c|c|c|c|}
\hline Alarm Condition & Alarm Name & $\begin{array}{c}\text { Alarm Limits } \\
\text { (Lower/Upper) }\end{array}$ & Priority & Type \\
\hline Heart Rate & HR HI/LO & $50 / 120$ & Warning & Parameter \\
Peripheral Oxygen Saturation & SPO2 HI/LO & $90 / 101$ & Warning & Parameter \\
Systolic Non-Invasive Blood Pressure & NBP S HI/LO & $90 / 150$ & Warning & Parameter \\
Diastolic Non-Invasive Blood Pressure & NBP D HI/LO & $30 / 90$ & Warning & Parameter \\
Median Non-Invasive Blood Pressure & NBP M HI/LO & $55 / 100$ & Warning & Parameter \\
Respiratory Rate & RSP HI/LO & $8 / 30$ & Advisory & Parameter \\
Tachycardia & TACHY & $\mathrm{n} / \mathrm{a}$ & Warning & Arrhythmia \\
Bradycardia & BRADY & $\mathrm{n} / \mathrm{a}$ & Warning & Arrhythmia \\
Ventricular Tachycardia $>2$ & VT > 2 & $\mathrm{n} / \mathrm{a}$ & Warning & Arrhythmia \\
Accelerated Ventricular Rhythm & ACC VENT & $\mathrm{n} / \mathrm{a}$ & Warning & Arrhythmia \\
Pause & PAUSE & $\mathrm{n} / \mathrm{a}$ & Warning & Arrhythmia \\
R (wave) on T (wave) & R ON T & $\mathrm{n} / \mathrm{a}$ & Advisory & Arrhythmia \\
Couplet & COUPLET & $\mathrm{n} / \mathrm{a}$ & Advisory & Arrhythmia \\
\hline
\end{tabular}

Table 1.3: An outline of the alarms within the scope of the project, along with their default settings at Hartford Hospital, is shown. See Appendix A for descriptions of the conditions associated with the arrhythmia alarms. 


\section{Methods}

\subsection{Alarm Data Collection \& Processing}

Unit alarm data was collected per device on a monthly basis, with the alarm histories being available on the first day of the month following its occurrence (i.e. March's data would be available on April 1, etc.). BedMasterEX assigned each device an identification number associated with its location in the hospital, which was also associated with a separate, arbitrary identifier, based on the number of reporting devices for that unit, which replaced the patient name and identification number for each alarm. This allowed for assurance of complete unit alarm history exports without risking violation patient privacy guidelines established in the Health Insurance Portability and Accountability Act (HIPAA) of 1996.

The total number of alarms per device was recorded in a summary spreadsheet for each month of data collection, along with the admitted and discharged hours given by BedMasterEX. Telemetry beds did not have this information, as patients are admitted or discharged from the CIC rather than the telemetry pack, so the online and offline hours were recorded as a substitute. Once all device alarm histories for a unit had been collected, they were compiled into a single unit alarm history. From this list, the frequency of each of the targeted alarms was determined using Microsoft Excel's "Find" function, and recorded along with the device alarm frequencies for that month. Running averages were kept for the alarms occurring on each of the units, but expressing these values as raw numbers of alarms did not allow for comparison beyond that unit. In order to more accurately express the number of alarms occurring based on the size of the unit, the raw number of alarms was converted to alarms per bed per day (A/B/D) (see Eq. 2.1). See 


$$
A B D=\frac{\text { Alarms }}{\text { Bed } \cdot \text { Day }}=\frac{(\text { Total Alarms })}{\text { No. of Beds in Unit }(\text { No. of Days of Unit Data Collection })}=\frac{A}{B \cdot D}
$$

Equation 2.1: The generalized calculation of alarms per bed per day is shown. The value for "Total Alarms" represents all alarms within the specified scope of the data set.

Appendix B for a description of various methods of expressing alarm frequencies based on available information.

With the unit data exported into Excel format, the desired information was available, but not in a condition that was conducive to analysis beyond counting, as each of the alarms would have to be analyzed manually. To better separate the information stored in each alarm instance a series of functions were developed in MATLAB (MathWorks, Natick, MA) and combined into a single graphical user interface (GUI). Because the MATLAB functions to read and write to Excel spreadsheets were slow to process unit alarm histories (taking upwards of 15 minutes per unit in some instances), and alternative format was required to process data efficiently.

The conversion of the spreadsheets was done using a standalone MATLAB script that converted the $1 \times 4$ array of strings representing each alarm instance into a $2 \times 6$ numeric matrix that could be stored in a text file. This mandated the development of a categorizing algorithm, which identified the alarms by source, the specific alarm condition, and the information associated with it (see Fig. 2.1). This configuration facilitated the use of sorting functions in the GUI to be able to isolate specific alarms or groups of alarms for further analysis, as well as allowed the unit alarm histories to be imported approximately 600 times faster than from Excel.

$\left.\begin{array}{cccccc|}\hline \text { Type } & \text { Condition } & \text { Priority } & \text { Duration } & \text { Parameter } & \text { Patient ID } \\ \text { Year } & \text { Month } & \text { Day } & \text { Hour } & \text { Minute } & \text { Second }\end{array}\right]$

Figure 2.1: A generalized version of the converted alarm event array is shown. Not that the type, condition, and priority elements of the array are coded as numeric values, though they represent strings. 


\subsection{Alarm Data Analysis}

Using the unit alarm summaries in Excel, the running averages for the number of alarms per bed per day of the targeted "Warning" and "Advisory" priority alarms were monitored as distributions both for the individual units and by acuity level. Graphs were designed to update as new information was entered into the spreadsheet. These graphs developed into alarm frequency distributions for each acuity level, identifying which alarms were most common at each acuity level. The unit distributions were also monitored to determine if there was any one type of unit that deviated from the average distribution of units at that acuity level.

The MATLAB GUI developed required an input created in BedMasterEX, which could either be the spreadsheet generated on data export, or the .txt file created in MATLAB. Once the file had been imported successfully, the user had two sets of options to manipulate. The first set, under the "Alarm Filtering" pane, was a series of menus the user could use to define a specific vital sign source and the desired alarm(s) associated with that source (see Fig. 2.2). The specified alarm(s) would be plotted on the adjacent plot window, showing the distribution curve for that alarm condition. Hartford Hospital's default alarm limits were imported, and the physiologically acceptable range was highlighted in green. When only looking at a single alarm (i.e. high heart rate or low $\mathrm{SpO}_{2}$ ), the completely physiologically acceptable range would not be shown, but a portion of it would be highlighted to show where the distribution fell in relation to it. The user would be informed of the percent of the total alarm history, both as a raw number as a percentage. The user would also be given control over the limits associated with the selected alarm(s). If the user chose to expand the alarm limit range, the GUI would determine which alarms would not have been activated, and highlight the corresponding area in yellow. Those alarms still outside of this new limit would be given red bars. To the right of the "Alarm 


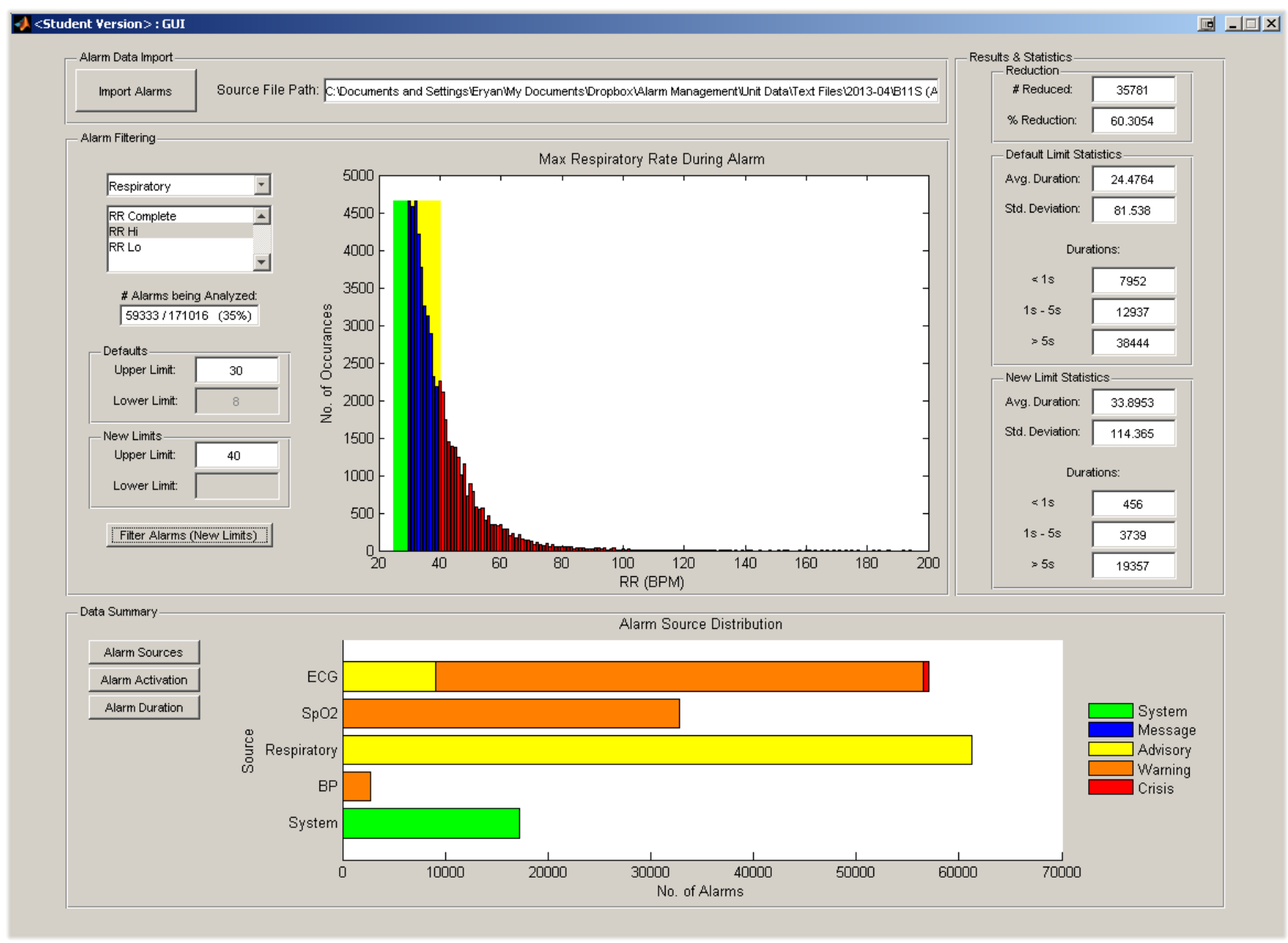

Figure 2.2: An example of the MATLAB GUI analyzing a set of unit alarm data is shown. The main window is showing the impact of limit changes to the distribution of high respiratory rate alarms, while the lower window is giving the distribution of alarms based on their source.

Filtering" pane was a results section that gave basic statistical information regarding the number of alarms reduced, and the impact on average duration of the alarms. The plot data from these experiments was copied and updated on the MATLAB workspace as the user interacted with the data, and was exportable at any time in the form of a vector appropriate to the plot size. These vectors were catalogued in spreadsheets according to their vital sign source, and used to develop condition distribution averages for the units, their acuity levels, and an overall average of all tracked units. 
A secondary function of the GUI was to give information regarding the time of day in which alarms were activated. Using the timestamp given with each alarm instance, the GUI would generate a minute-to-minute histogram of the alarms in that imported file. Alarm duration was also taken into account, with alarms that extended over a minute in duration being included in the next minute's total number of alarms. As with the data from the "Alarm Filtering" pane, this histogram's plot data was exportable as a vector, and was stored for each unit in a spreadsheet. From there, the data used was compiled to create unit average, acuity level average, and a general histogram for all tracked units. The plot data from these two methods served as the primary source of data for the analysis performed. 


\section{Results \& Discussion}

Over the course of the eight-month sampling period, a total of 9.76 million alarms were observed across the twelve tracked units. This represents an average of 295.86 alarms per bed per day, or one alarm being activated once every five minutes for the duration of the study. See Appendix $\mathrm{C}$ for the alarms per bed per day monthly averages for each of the tracked units, as well as their eight month average. Of those 9.76 million alarms, 7.65 million $(78.5 \%$ of the total alarms catalogued) were part of the target subset, being generated by the identified subset of "Warning" and "Advisory" priority alarms. These alarms were made into multiple sets of distributions, with these distributions being replicated at the unit level, acuity level, and as a hospital-wide average from the tracked units.

\subsection{Physiological Alarm Frequency Distributions}

The first set of distributions developed from the data collected was to determine the most common alarms at each acuity level, and to determine the source of changes in alarm frequencies as either a result of changes in the types of parameters monitored, or changes in the physiological behaviors of the patients. Results were expressed as percentages in order better relate the prevalence of a given alarm, as opposed to in alarms per bed per day (the values used to determine the percentages), which does not give as much context to the frequency of an alarm at a given acuity level. All alarms, physiological and technical, not represented in the target subset of "Warning" and "Advisory" priority alarms were grouped into the category labeled "Other." See Appendix D for the breakdown of how the alarm frequency distributions of individual units contributed to the acuity level averages. 
The three Med/Surg telemetry units generated a total of 4.12 million alarms over the data collection period, averaging 123.94 alarms per bed per day. These units monitored the least number of vital signs, focusing solely on the ECG waveform and its associated parameters. As a result, this limited the number of possible alarms that could be triggered (see Fig. 3.1). Couplet alarms were the most prevalent $(24.26 \%$ of all alarms observed), with alarms pertaining to high and low heart rate being the next most common. The remainder of the arrhythmia alarms comprised a smaller percentage $(9.09 \%)$ than all but the bradycardia (BRADY) alarm. There was little deviation between the unit distributions, with alarm frequencies differing by less than $1 \%$ for all categories (see Appendix D).

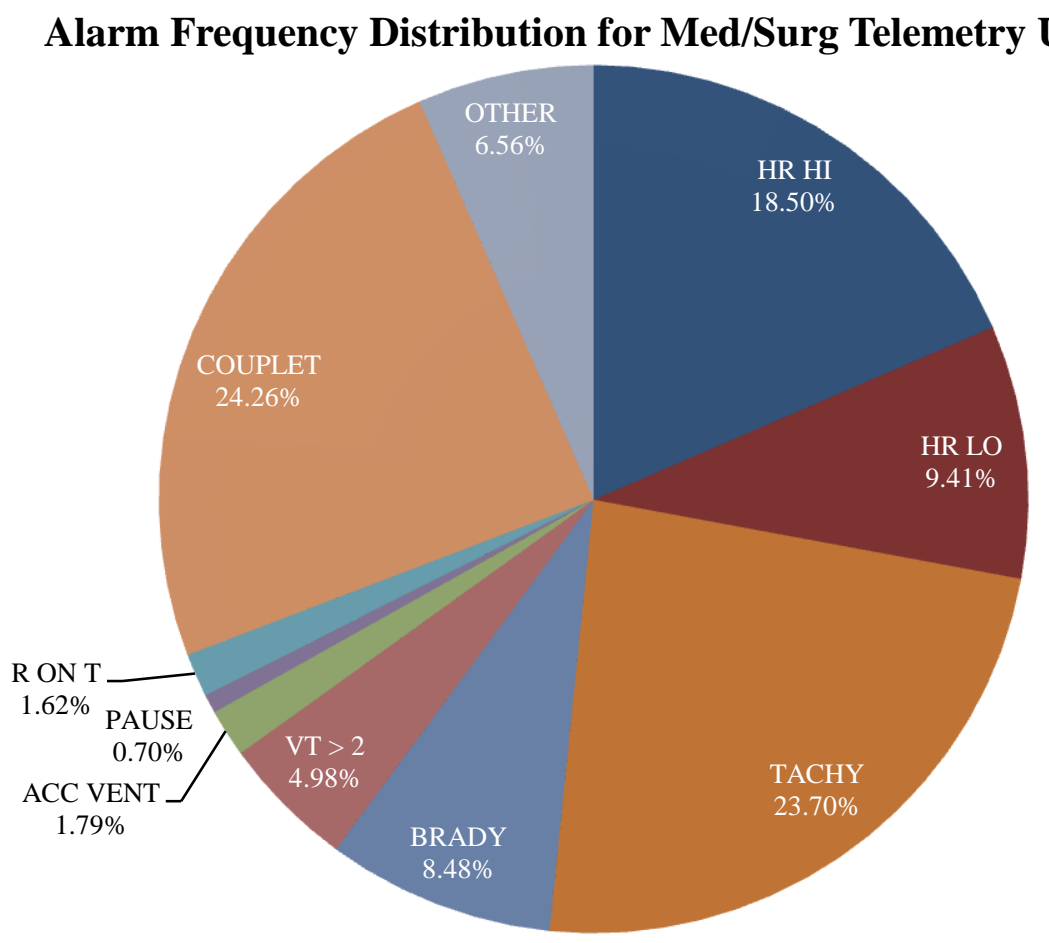

Figure 3.1: The alarm frequency distribution for the tracked Med/Surg units is shown. Possible alarms are limited to those with the ECG as a source and technical alarms. 


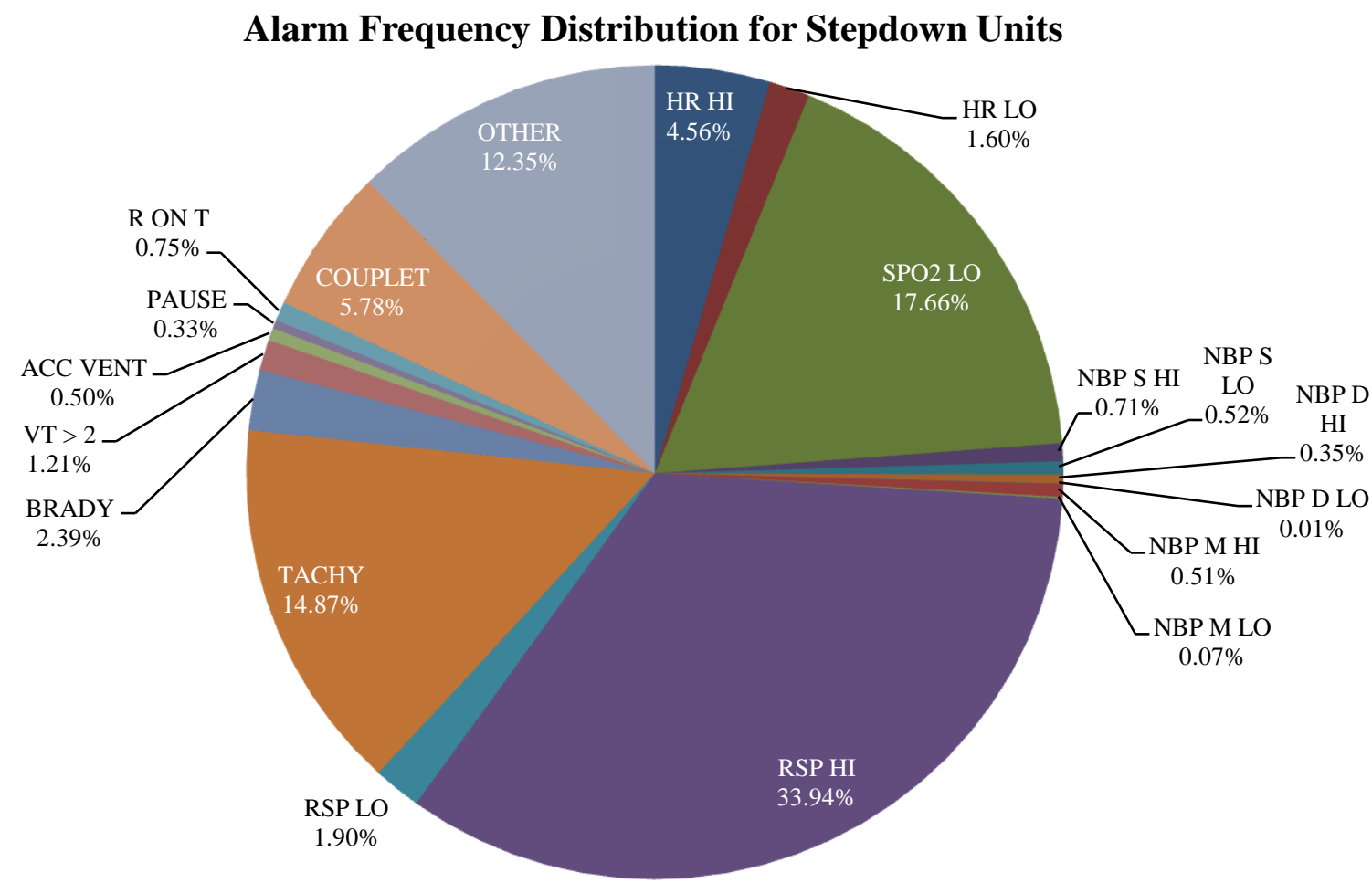

Figure 3.2: The alarm frequency distribution for the tracked Stepdown units is shown. Monitoring at this acuity level expands to include pulse oximetry, respiratory monitoring, and in some cases, invasive blood pressures.

The Stepdown units generated 2.59 million alarms across the four tracked units, equivalent to 366.95 alarms per bed per day. Using SOLAR 8000i monitors, these units monitor a wider range of parameters than the telemetry units; incorporating pulse oximetry and respiratory monitoring into their regularly monitored vital signs (see Fig. 3.2). High respiratory rate alarms were the single greatest contributor, with the alarm making up more than one third of the total alarms for all but the Neurosurgical Stepdown unit, where it made up only $7.67 \%$ of the total alarms. Besides this deviation, the neurosurgical Stepdown still showed similar alarm prevalence to the other Stepdown units, with high heart rate, low $\mathrm{SpO}_{2}$, and couplet alarms having the highest frequency (see Appendix D). 
ICUs contain the highest acuity patients, and require the greatest level of monitoring. This resulted in an increase in alarms that were considered out of scope of the study, particularly those pertaining to invasive pressure monitoring (see Fig. 3.3). Strictly examining the alarms within the scope of the study, the alarm frequency distribution resembles that of Stepdown units, with high respiratory rate, high heart rate, and low $\mathrm{SpO}_{2}$ being the most common alarms.

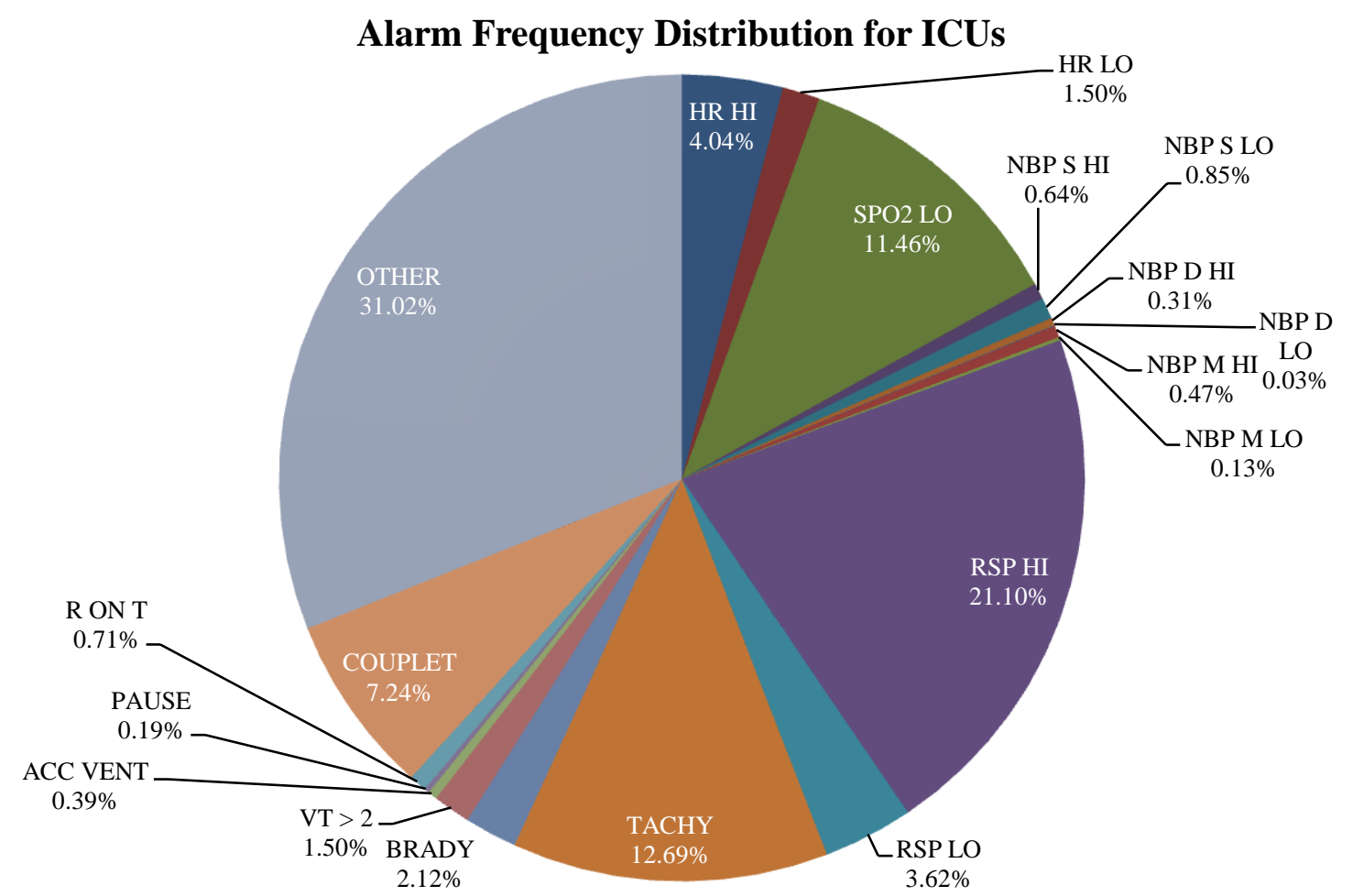

Figure 3.3: The alarm frequency distribution for ICUs is shown. There is an increase in out of scope alarms primarily due to the increase in use of invasive pressure monitoring.

These alarm frequency distributions confirm existing studies that identify parameter alarms for heart rate and $\mathrm{SpO}_{2}$ as being prone to nuisance alarms, along with the couplet arrhythmia alarm $[7,11]$. However, respiratory rate alarms have not been specifically identified in previously published literature, and, based on their prevalence in both the Stepdown unit and ICU distributions, merit further investigation as to their default settings. It is important to note that the traditional method for determining respiratory rate, using impedance measurement from 
the ECG leads, makes the measurement prone to error due to signal artifact, an issue that cannot be addressed by monitor settings.

These distributions, including the hospital-wide average (see Fig. 3.4) are necessary for determining the percent alarm reduction for the target area. Using the percentage appropriate to the unit (or the acuity level if unit specific data is unavailable), the reduction in that specific alarm can be expressed as to how it impacts the area in which the change was made, be it unit, acuity level, or a hospital-wide change in settings or policy.

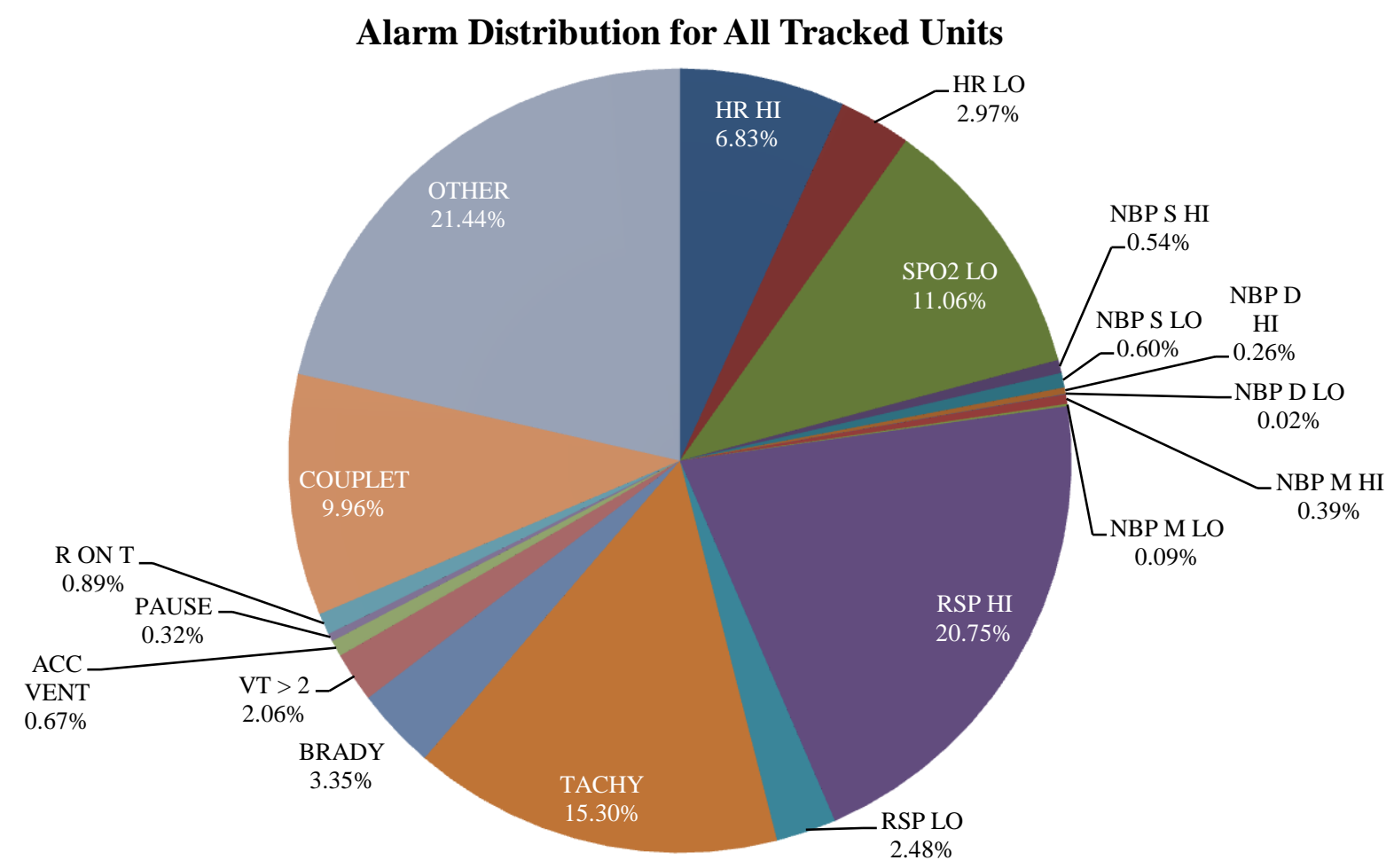

Figure 3.4: The alarm frequency distribution for all tracked units is shown. It most closely resembles the distribution seen for ICUs, but in general does not correlate well to any specific acuity level.

An important correlation to note in these distributions is the relationship between the high and low heart rate alarms and the tachycardia/bradycardia arrhythmia alarms. These alarms are near-duplicates of each other, with the only difference being that the arrhythmia alarms have an inherent three second time delay, while the parameter alarms have a five second delay. For 
events lasting greater than five seconds, this results in two alarms sounding for the same event, while offering no additional clinical benefit.

By changing the tachycardia and bradycardia alarms to "Message" priority, thereby removing the audible component of the arrhythmia alarms, events where the patient's heart rate exceeds the established upper heart rate limit will still be recognized, but the duplicate alarms will be removed. This results in an immediate reduction in the total number of alarms for each monitored unit, with telemetry units receiving the greatest benefit at a $32.18 \%$ reduction in alarms, Stepdown units at $17.26 \%$ reduction, and ICUs seeing the least benefit at $14.81 \%$ reduction. This duplication of alarms has been recognized by GE's patient monitoring division, and is to be addressed in future software upgrades for their newest generation of devices: the Carescape B Series monitors.

\subsection{Alarm Condition Distributions}

Separate distributions were developed for high heart rate, low heart rate, low $\mathrm{SpO}_{2}$, and high respiratory rate alarms based on the condition at alarm initiation. These were plotted as percentages of the sample, with the intent of identifying the general shape of the distribution curve and any sort of abnormalities that may be a factor when determining default settings for that parameter. These distributions were made for each tracked unit, compiled and remade for each acuity level, with these then being compiled again to develop a hospital-wide average for each of the alarms. This hospital-wide average was developed in order to maximize the sample size to minimize any spiking in the alarm frequencies at specific values, as this may have suggested patterns that may have been inherent in the patient population observed, but not of the system itself. 


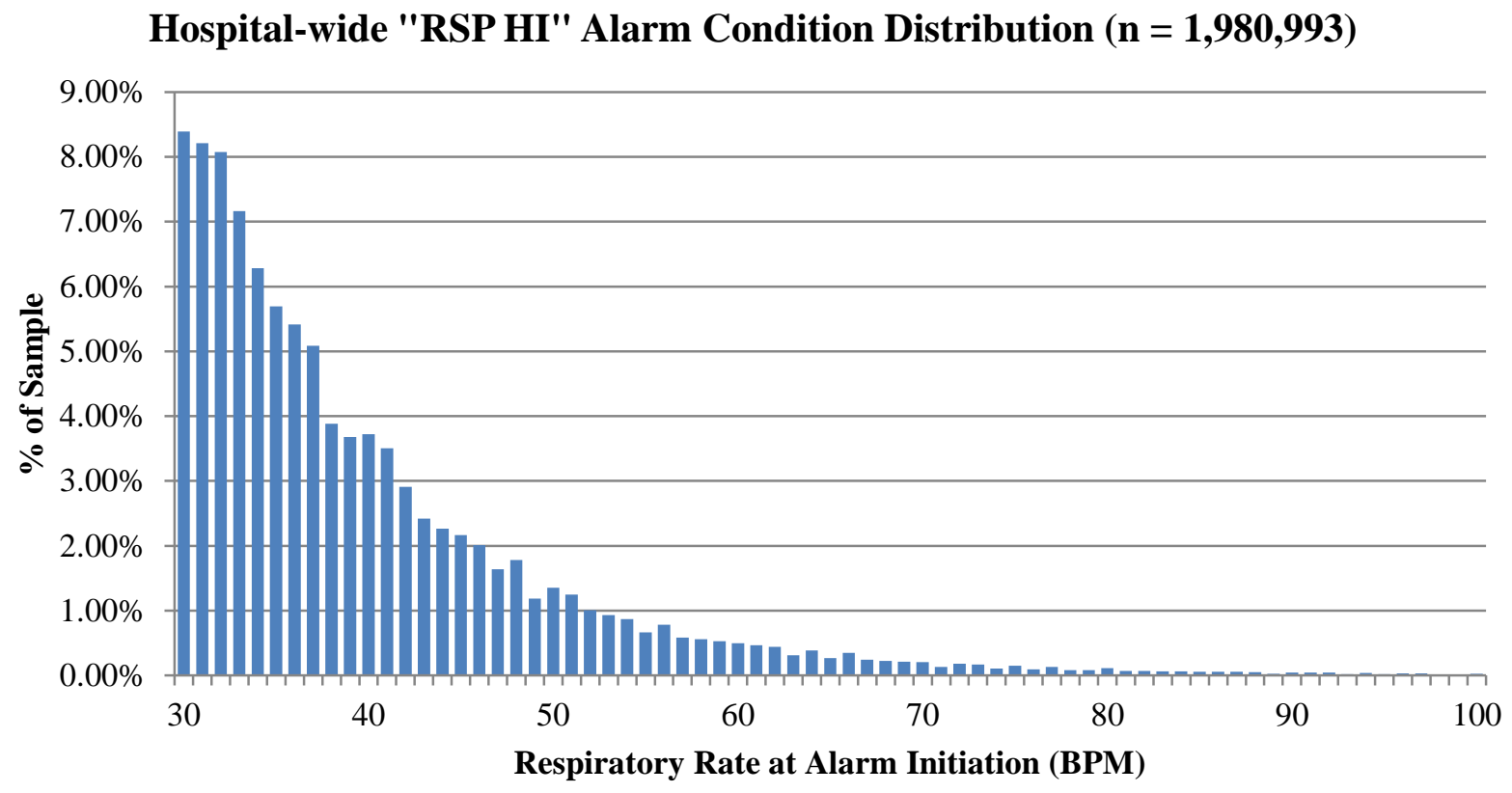

Figure 3.5: The alarm condition distribution for high respiratory rate alarms across all tracked units is shown. Note that only ICUs and Stepdown units reported this type of alarm.

High respiratory rate alarms, being the single largest contributor to the hospital's total patient monitoring alarms, exhibited a distribution curve similar to what has been seen by Taenzer et al. [13]: exponential decay as the condition moves farther away from a physiologically acceptable range (see Fig 3.5). Though not a perfect exponential decay distribution, there is nothing notably unusual about the high respiratory rate alarms. Because of the high frequency of occurrence, recommendations for this alarm would be to determine if the limit set for the facility is too tight and warrants expanding.

Low $\mathrm{SpO}_{2}$ alarms represented a much more rapid decay than the high respiratory rate alarms, with $89.89 \%$ of the alarms initiated between $80 \%$ and $90 \%$ oxygen saturation (see Fig. 3.6). This shows that adjustments to the alarm limit can result in large reductions in the total number of low $\mathrm{SpO}_{2}$ alarms. Dartmouth Hitchcock Medical Center (Lexington, NH) has made the largest change to date, moving their low $\mathrm{SpO}_{2}$ limit to 80\% [7], though this has been met with some skepticism and concern, especially if this were to be implemented at higher acuity 
levels. The more conservative limit change proposed by Graham et al., shifting the limit to $88 \%$, has been met with greater acceptance.

High heart rate alarm limits have traditionally been set at multiples of 10 , whether by default, or after analysis for alarm reduction $[11,12]$. However, the distribution obtained from

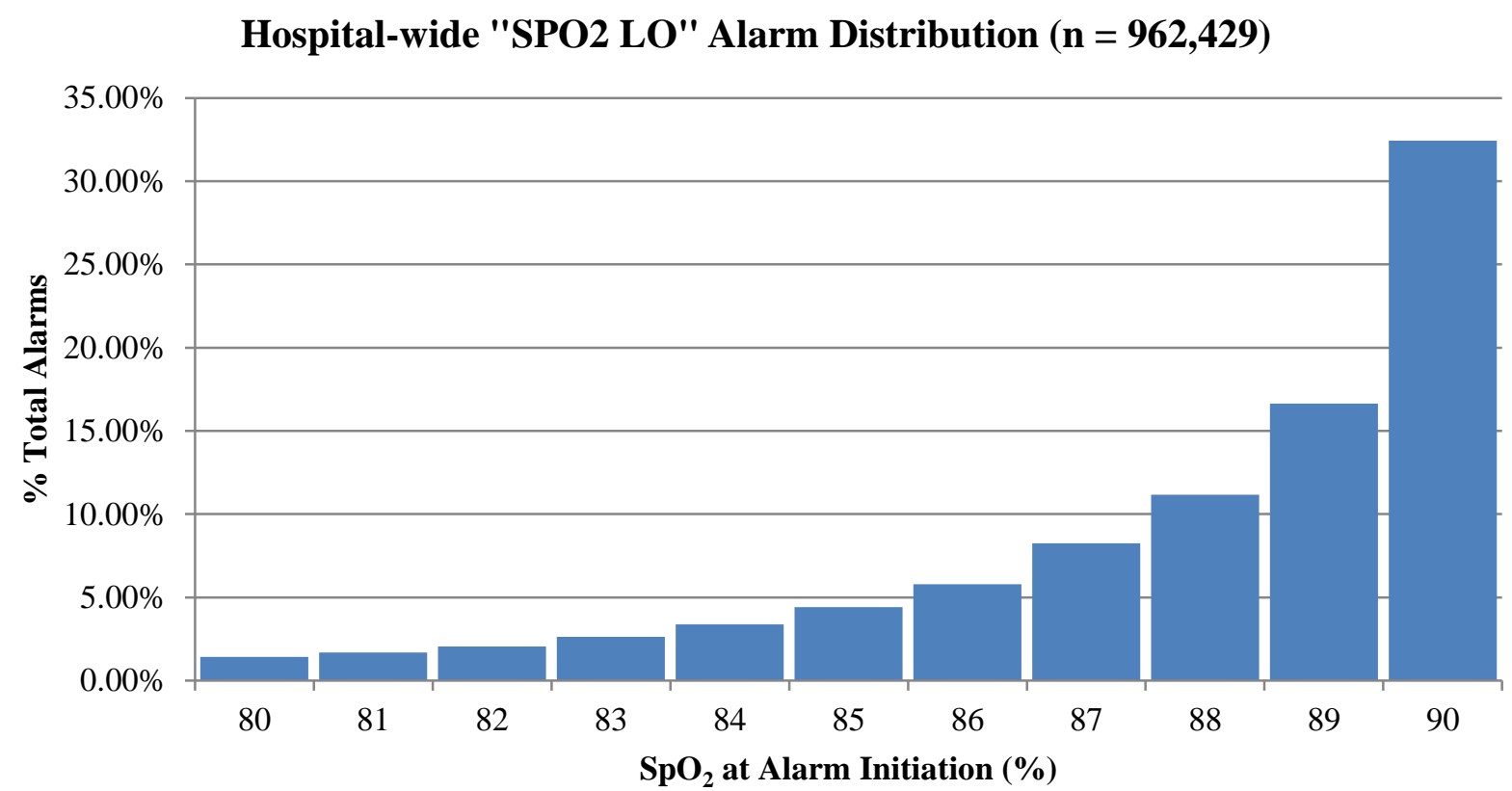

Figure 3.6: The upper portion of the alarm condition distribution for low $\mathrm{SpO}_{2}$ alarms across all tracked units is shown. Not that only ICUs and Stepdown units reported this type of alarm.

each of the tracked unit shows an abnormality not observed in either the respiratory rate or $\mathrm{SpO}_{2}$ alarm condition distributions (see Fig. 3.7). The high heart rate distribution does resemble an exponential decay curve, however, this decay repeats over each heart rate that is a multiple of 10 , with an increase in the number of alarms activated at each multiple. This pattern became more apparent in the unit alarm distributions as the acuity level increased. Low heart rate alarms showed a similar pattern to that observed with high heart rate alarms, but with the repetition occurring at heart rates that were multiples of 5 (see Fig. 3.8).

These patterns in heart rate distributions present an additional consideration that needs to be made when determining heart rate alarm limits. If deemed clinically safe, as opposed to using 
heart rate that is a multiple of 10 for the upper heart rate limit, the limit should instead be set to one greater than that multiple of 10 (i.e. 131 instead of 130) in order to eliminate the spike in alarm frequency occurring at that condition. The same concept applies to the lower heart rate limit, where, if deemed clinically safe, the limit should be placed at one below a multiple of 5 (i.e. 44 instead of 45). Is used, this methodology also requires education of the clinical staff that has been given the authority to adjust patient monitoring alarm settings in order for any changes to be optimized for the system.
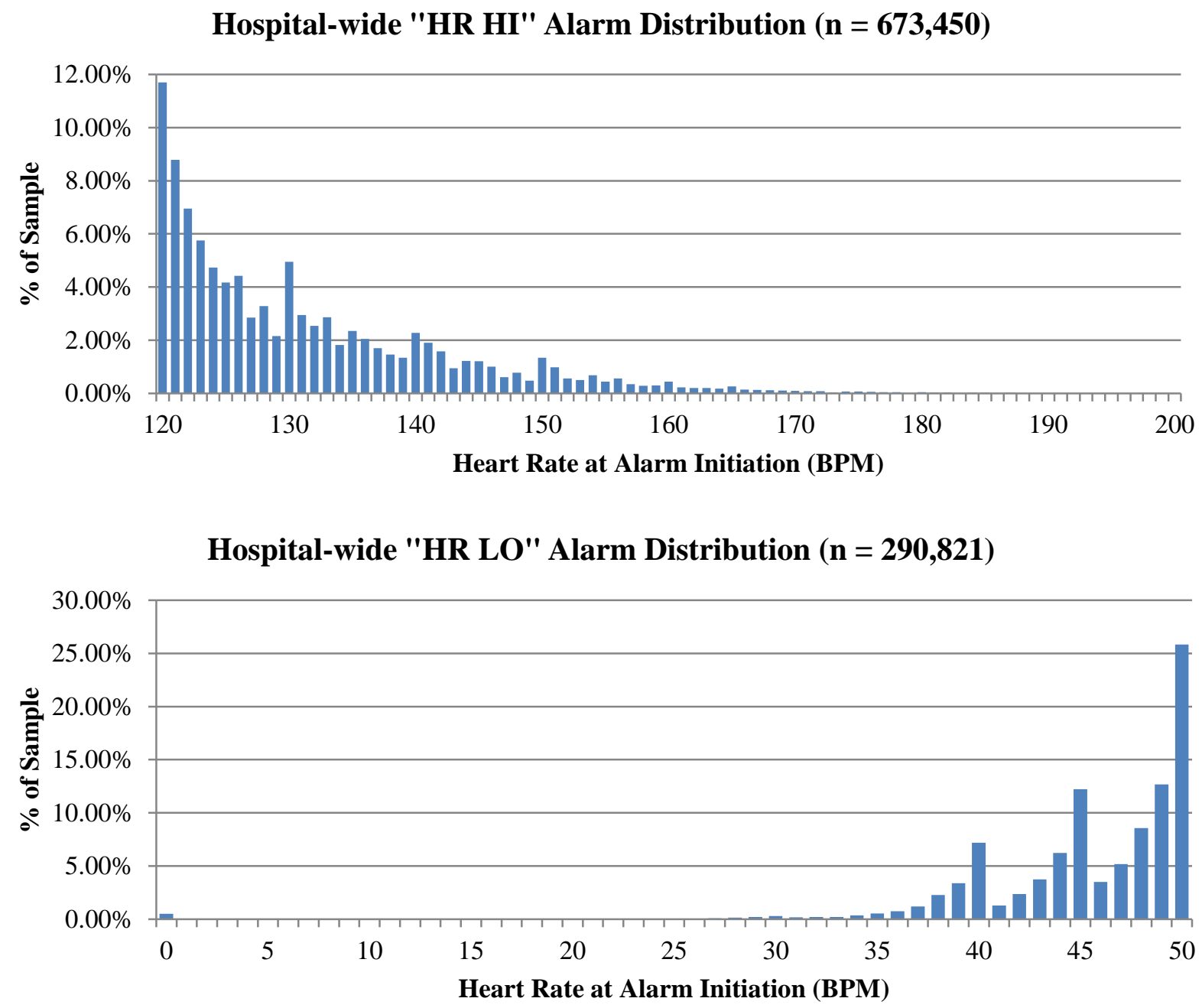

Figures $3.7 \&$ 3.8: The alarm condition distributions for high and low heart rate, respectively, across all tracked units is shown. Note the repeating exponential decay pattern occurring at each multiple of 10 in high heart rate, and each multiple of 5 in low heart rate. 


\subsection{Comparing Coefficients of Determination $\left(\mathbf{R}^{2}\right)$ Across Acuity Levels}

The condition distributions described are based on an average of all tracked units reporting the specified alarms. This does not, however, explain differences or patterns that may be inherent to certain specialties or acuity levels, be it due to patient behavior, workflow, or the monitoring technology used in each area. In order to quantify the comparisons made, the coefficient of determination, $\mathrm{R}^{2}$ (see Eq. 3.1), between the unit averages, the acuity level averages, and the hospital-wide average were calculated. The coefficient of determination uses a scaling of 0 to 1 to define the correlation between data sets, where the greater the calculated $R^{2}$ value, the greater the similarity between the two compared sets of data. In order to avoid the possibility of having a high calculated value misrepresent the similarity of the distributions, a qualitative visual inspection was included in order to ensure that the general shape of the distribution curves were appropriate to the $\mathrm{R}^{2}$ value calculated. [14]. By combining these quantitative and qualitative tests, areas of significant deviation from the established average could be identified, and the degree of differentiation could also be established. See Appendix F for a complete list of calculated coefficients of determination for the alarms analyzed.

$$
R^{2}=\frac{\sigma x, y^{2}}{\sigma(x) \cdot \sigma(y)}
$$

Equation 3.1: The calculation of the coefficient of determination is shown. In this circumstance, $\mathrm{x}$ and $\mathrm{y}$ refer to separate sets of data. The greater the calculated $\mathrm{R}^{2}$ value, the greater the similarity between data sets $\mathrm{x}$ and $\mathrm{y}$.

It is important to note that the coefficients of determination calculated are based on the unit to hospital-wide average, as the number of contributing acuity level averages can lead to distributions that yield very high $\mathrm{R}^{2}$ values. By comparing to individual units, this allows for better identification for variations that may be unique to a given specialty, or specific unit. 
The high respiratory rate alarm condition distributions showed high coefficients of determination for all comparisons made, with the distribution curves only deviating from each other at the lowest end of the curves (see Fig. 3.9). Of the four alarms analyzed, these distributions showed the greatest correlation to each other, with an average $\mathrm{R}^{2}$ value of 0.998 , and with the lowest calculated $\mathrm{R}^{2}$ value being 0.994 . The coefficients of determination calculated for the low $\mathrm{SpO}_{2}$ alarm condition distributions showed slightly lower correlation than the high respiratory rate alarm condition distributions, averaging 0.991 , with the lowest being 0.946 (see Fig. 3.10).
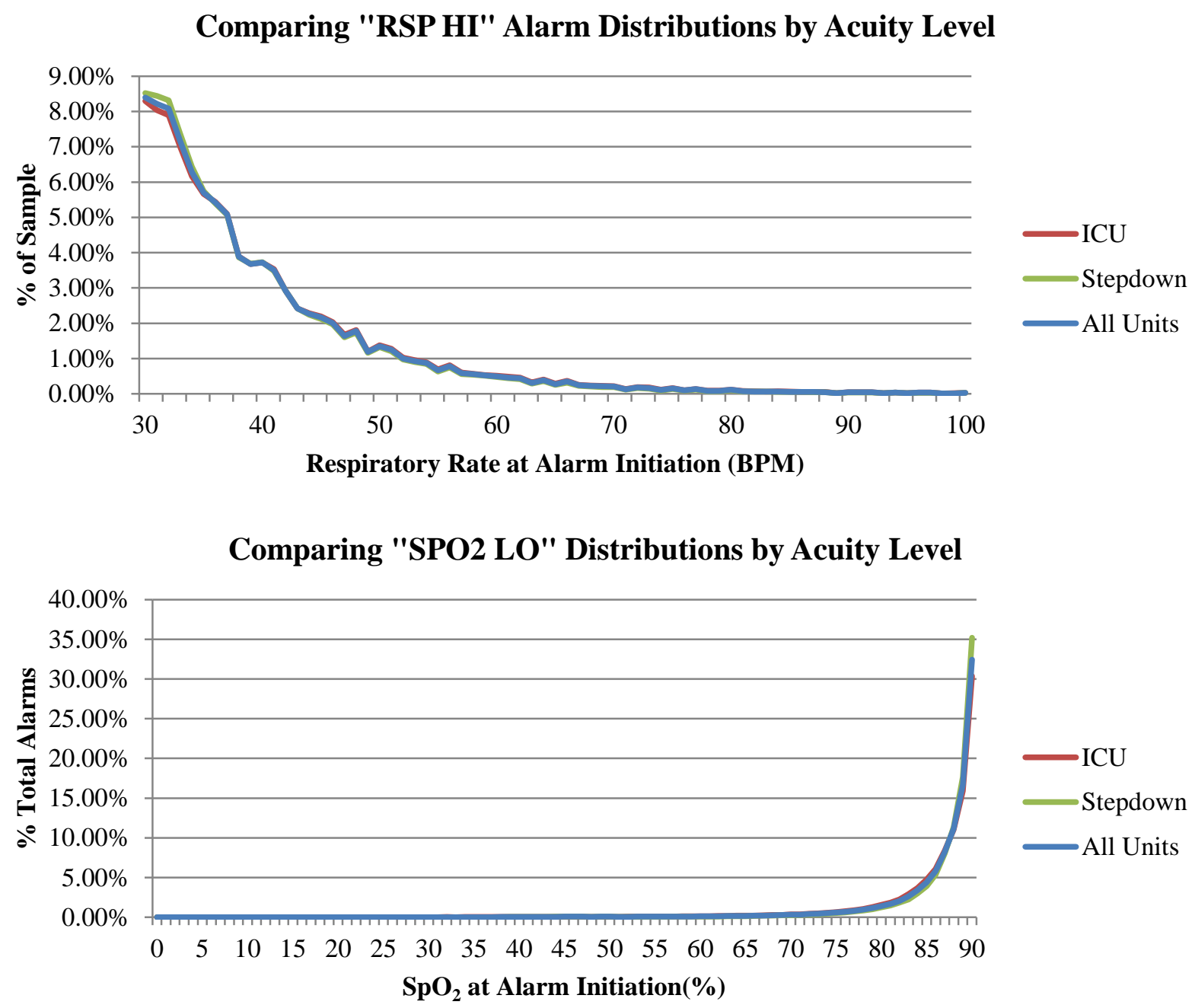

Figures 3.9 \& 3.10: The comparisons between distribution curves for individual acuity levels and the hospital-wide average for high respiratory rate and low $\mathrm{SpO}_{2}$ alarms, respectively, are shown. 

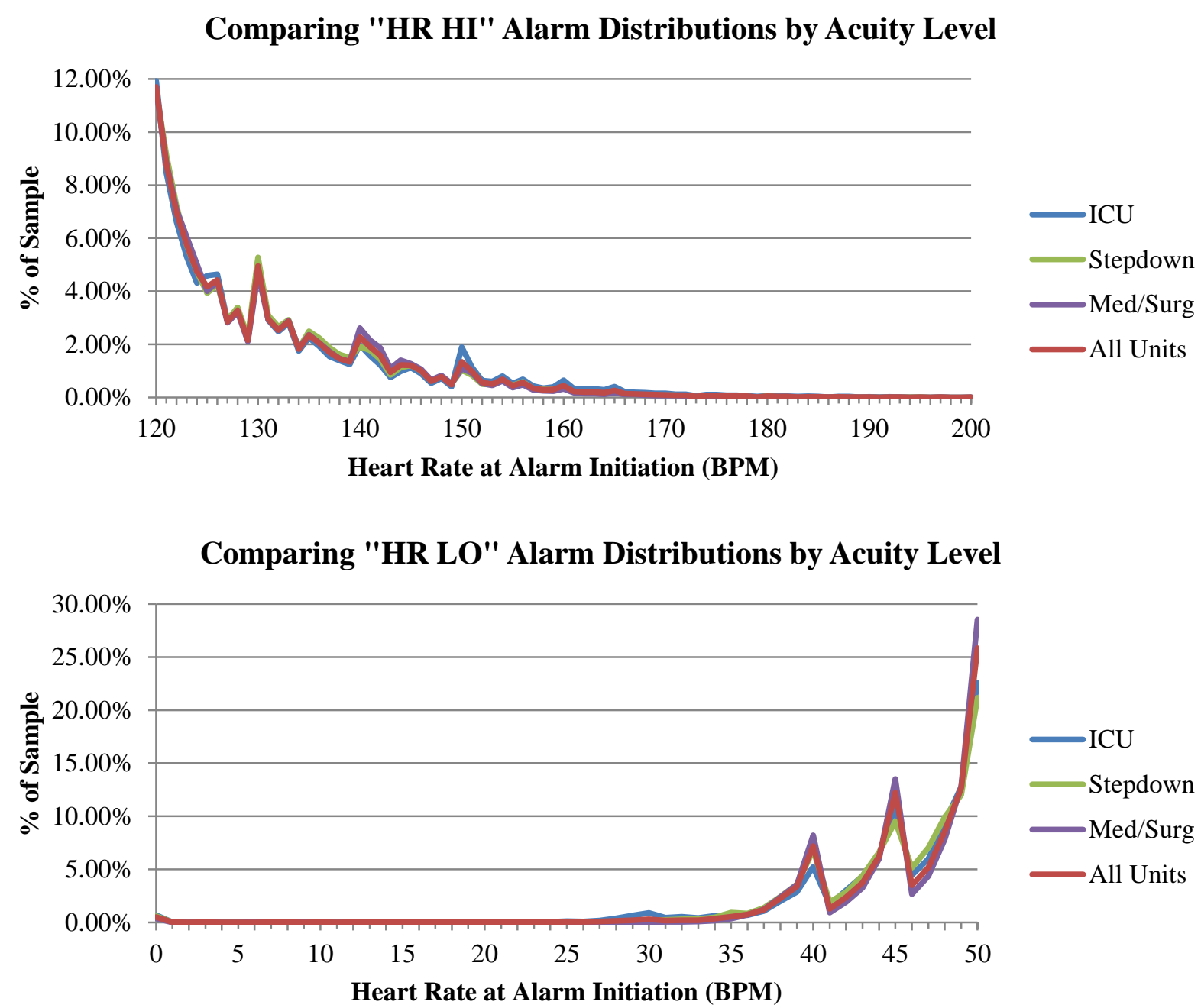

Figures $3.11 \&$ 3.12: The comparisons between distribution curves for individual acuity levels and the hospital-wide average for high and low heart rate alarms, respectively, are shown.

The heart rate alarm distributions show visibly greater deviation from the hospital-wide average, particularly in the areas of each distribution curve where the frequency of alarm spiked. For high heart rate alarms, there was no acuity level that consistently deviated (see Fig. 3.11), while for low heart rate alarms, the Med/Surg unit distribution appeared to be the most sensitive, consistently having the lowest percentage frequency of alarms after a spike occurred, and the highest percentage frequency during the spikes (see Fig. 3.12). That being said, the coefficients of determination calculated were still high. For high heart rate alarm distributions, the average $\mathrm{R}^{2}$ 
value calculated was 0.969 , with a minimum of 0.891 , and the low heart rate alarm distributions exhibited an average $\mathrm{R}^{2}$ value of 0.928 , with a minimum value of 0.781 from the Neurosurgical Stepdown unit.

The data collected for the alarm frequency distributions shows that the number of alarms per bed per day seen by a unit varies significantly with acuity level. However, when looking at the distribution of those alarms based on their condition, the distributions do not show the same degree of variation. On the contrary, distributions from units of other acuity levels, when shown as percentages, can predict the alarm behavior of other units outside of its specialty and acuity level. This presents an opportunity to decrease the total amount of baseline data collection necessary to determine the impact of various interventions to reduce the number of alarms. This is particularly important in facilities where it is difficult or impossible to obtain information from specific units or acuity levels. Although having baseline data native to each unit in the facility would be ideal, this data suggests that an accurate estimate can be obtained by looking at data from other locations within the facility. It also supports the idea of developing hospital-wide default settings, if deemed clinically safe, as these changes will have very similar impacts to the percentage of alarms reduced in each unit.

The only exception to this appears to be neurosurgical units, as both in the alarm frequency distributions and the alarm condition distributions, units of this specialty appeared to deviate from the other units tracked. Further investigation is required to determine what physiological factors may impact this, as well as changes in workflow due to the types of patients in these units. 


\subsection{Clinical Alarm Distribution by Time of Day}

Using the timestamps for each of the alarm instances, accompanied by the durations for those alarms, the number of active alarms at a given time was determined for each unit. These distributions were made to describe the unit's daily timeline of active alarms. These distributions were compiled based on acuity level and plotted. By examining these distributions, the goal was to identify what times of day have the greatest likelihood for alarms to be triggered. Using this information, conclusions could be drawn as to potential causes for the increase in alarms. The metric for these distributions, alarms per day, is arbitrary. The value means nothing on its own, but rather serves as a means of comparison to other times in the day. It does not correlate between acuity levels, as the value does not account for unit sizes or patient load in any way.

All three acuity levels exhibited a spike in alarm activation starting at approximately 7am, which corresponds with the change of shift on the units. This spike peaks at approximately 9am, which also corresponds with breakfast and rounding of physicians. After this spike, the units begin to differ in behavior based on acuity level. On the Med/Surg units, the morning increase in alarm activation returns to a baseline slowly over the course of the day (see Fig. 3.13). There is a rise in alarm activation around $2 \mathrm{pm}$ and $6 \mathrm{pm}$, which can be related to several factors, including additional rounding, meals, and visitors.

Stepdown and ICU patients experience more regular rounding than patients on telemetry units, and, in general, are considered to be less stable. For Stepdown patients, after the initial spike at 7am, the alarm activation rate decreases some, but shows some spiking corresponding with the top of the hour, a trends which lasts until approximately 8pm (see Fig. 3.14). This spiking is more clearly visible for ICU patients, who consistently exhibited the hourly spiking throughout the day and into the night (see Fig. 3.15). Both acuity levels also showed a rise in 
active alarms beginning at approximately 11:30pm, and lasting until 2am, where the number of active alarms drops to a much lower level by 3am. The cause(s) of this anomaly are currently under investigation.

Active Alarms by Time of Day for Med/Surg Units

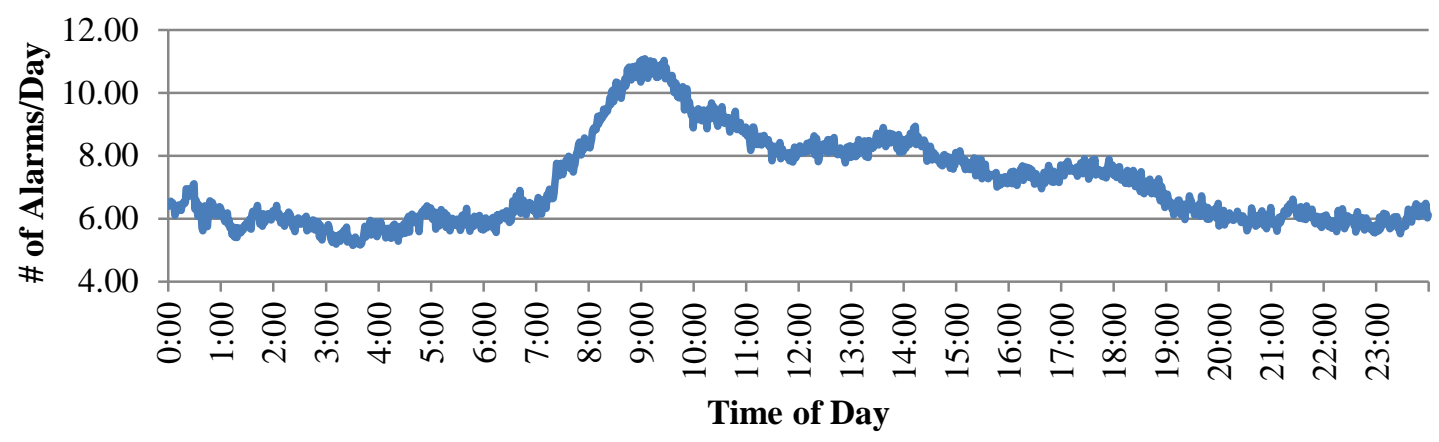

Active Alarms by Time of Day for Stepdown Units

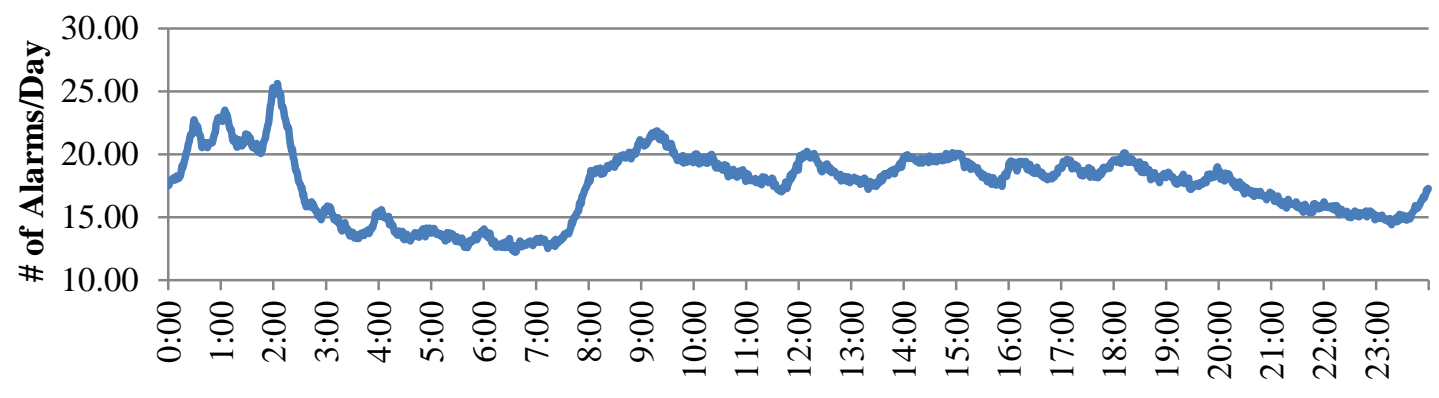

Time of Day

Active Alarms by Time of Day for ICUs

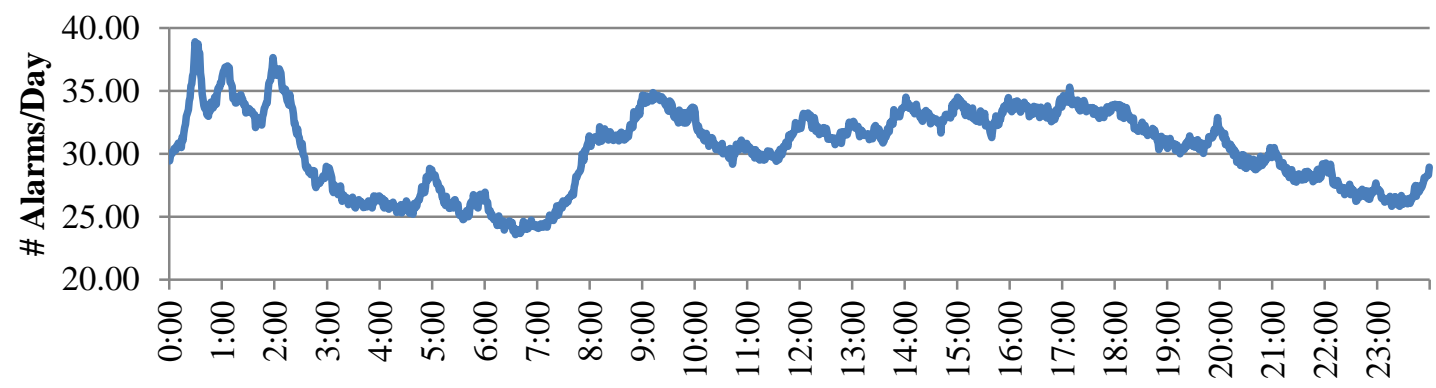

Figures 3.13-3.15: The distribution of active alarm by time of day for each acuity level is shown. Spikes in active alarms were correlated with regular activities on the unit, aiming to better understand differences in unit workflow. Note that as acuity level increases, so does the sensitivity of the patients to disturbances. 


\subsection{Sources of Error \& Mitigation Strategies}

Though the large sample size of this study was meant, in part, to generate a view of the "average" patient and their potential physiological behavior, several sources of error needed to be identified and to some degree qualified in order to ensure that the data being collected remained viable.

Unlike many studies, the population for this study was constantly in flux. The sample is inherently randomized, as there are no controls in place to dictate exactly who is being admitted to the hospital. Some patients do formally schedule their stay based on the availability of their doctor(s), but others arrive unexpectedly. There is some bias inherent to the population as well, as the hospital has different reputations for different specialties, and as a whole, the patient population shows heavier representation of the elderly. This, however, is representative of the adult hospital environment, and is not considered to have had any significant impact on the information collected.

Though plans of care are individualized to meet the needs of each patient, this does not necessarily correlate to the stability of their vital signs. Some patients will, inevitably, generate more alarms than others, and this cannot necessarily be predicted or managed immediately (or at all, in some cases). This can result in high concentrations of specific alarms at certain times. This is primarily mitigated by the study's sample size, and as such, is considered to have minimal impact on the information collected.

Technical sources of error impacted the data collection process at multiple points. The monitors collecting the data showed unexpected trends in behavior over the course of the study, particularly in terms of appearing to favor certain values for various conditions. This appeared in multiple cases, though the most obvious one appears to be in the distribution of the low heart rate 
(HR LO) alarms, where there are clear spikes in the frequency of alarms that occurred at heart rates of 45 and 40 beats per minute. This behavior is believed to be technical, as there is no published evidence suggesting a physiological predisposition to very specific heart rates. This, however, cannot be confirmed due to patent and copyright issues. This is considered a significant source of error, but appears to be consistent across all of the data, which lessens its impact. It also represents the expected experience of other facilities using similar patient monitoring equipment, and may provide insight into selecting new alarm limits.

The largest source of error identified in the study was the server storing the alarm data, more specifically its downtime. The server would unpredictably crash, and would result in alarms not being catalogued for the duration of the outage. The end result of this is that the alarm frequencies for each sampling period (one month) may be artificially low. That being said, all units would experience the same outage at the same time, which means that the alarm frequencies are universally artificially low. Of the sources of error identified, this had the most significant impact, and it is not possible to quantify the extent to which this impacted the data, as the alarm histories for those outages are not obtainable. 


\section{Conclusion}

The baseline data collected from this study has already been integrated into some of the first interventions suggested by Hartford Hospital's Alarm Management Committee. The data is serving as the primary means of illustrating the current state of patient monitoring alarms in the hospital and is being presented to the groups with the clinical authority to approve the alarm default changes. The method of data collection and filtering proposed in this study is also being used to monitor changes on the units as they are made, allowing for detailed feedback to participating units.

This study developed an initial profile of the patient monitoring alarm situation at Hartford Hospital, and yielded data that allowed for trends to be identified in the relationship between alarms observed at different acuity levels, as well as traits unique to alarms associated with specific vital signs. This information can provide general guidance when looking at changing alarm default limits. The next major step being pursued with this information is validation testing of the distributions presented for the alarms with numeric conditions. If the percentages described in each distribution are found to be adequately accurate, then alarm management committees will have an additional resource to determine their expected level of alarm reduction, including setting specific reduction goals.

From the perspective of patient monitoring alarms, there is still much that can be done with the information collected. The alarm data archived can be expanded upon, if the need is identified. The alarm histories also contain data for alarms deemed to be outside of the scope of this investigation. In particular, examining the limits set for ST measurements of the ECG waveform and invasive pressure monitoring may prove beneficial. This study also left technical alarms unexplored. All technical alarms are included in the alarm histories, and although there 
are not parameters that may be changed for these alarms at this time, the average frequency of these alarms can be used for determining the impact of other interventions to reduce the number of technical patient monitoring alarms.

Another area of consideration not addressed by this study is signal artifact generating false alarms. Artifact is typically an issue associated with the ECG and decisions made based on it. This includes all arrhythmia alarms, heart rate alarms, and respiratory rate alarms. The issue is generally attributed to the electrodes, with contributing factors ranging from build-up of sweat, oil, and other secretions increasing electrode impedance to the gel on the electrode drying out due to poor storage or extended use. Again, the baseline data collected in the study can be compared against data collected after an intervention to either change electrode products or change to policies and/or procedures regarding their storage, application, and replacement, the latter of which has been shown to reduce the frequency of several different alarms [11].

Alarm fatigue is not a problem exclusive to patient monitoring; there are other contributors to the overall issue. Many of these are other medical devices, while other sources are not clinical in nature (i.e. fire alarms). Some of these sources may be identified as needing no modification, while others may require similar data collection to that described for the patient monitoring alarms. In the latter case, some devices (such as ventilators and infusion pumps) may be integrated into the patient monitoring data stream, and have their alarms and notifications archived as part of the alarm histories. Expanding the alarm inventory in the MATLAB scripts will allow for these alarms to be filtered based on the identified need of the users, and similar analysis may be performed.

The methods proposed in this study are not designed to create sweeping changes in policy or clinical workflow. Rather, they are meant to be a series of small, progressive alterations that 
can be adapted as necessary to best suit the patient's needs. The overall goal of alarm management is to provide a baseline of settings and processes to mitigate false and nuisance alarms, while providing a toolkit to the clinical staff to optimize this configuration based on each patient's needs. This is a living process, and hospital policy will need to be adapted as the technology changes in order to ensure patient safety and proper system usage. 


\section{Appendices}

\section{Appendix A - Clinical Definitions of Select Cardiac Arrhythmia Alarms}

The definitions below are the published definitions by GE Healthcare of the arrhythmia alarms considered to be in the scope of this study [12]. The alarms are listed alphabetically and any referenced values are those assuming the patient is an adult. Pediatric and neonatal patients have different numerical thresholds for these alarms.

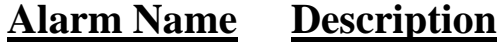

ACC VENT Accelerated ventricular occurs when six or more ventricular beats are detected with an average heart rate for the ventricular beat between 50 and 100 beat per minute.

BRADY Bradycardia is the average of the most recent eight $\mathrm{R}$-to- $\mathrm{R}$ intervals at a heart rate less than the set LOW heart rate limit.

The BRADY limit matches the low heart rate limit that is set under the Parameter Limit alarms. If the low heart rate limit is changed, the BRADY limit changes.

COUPLET Occurs when two ventricular beats are detected and have nonventricular beats before and after the couplet. The coupling interval must be less than 600 milliseconds.

PAUSE Occurs when a 3-second interval without a QRS complex is detected.

R ON T Occurs when a ventricular complex is detected within the repolarization period of a non-ventricular beat.

TACHY Tachycardia is four R-to-R intervals at a heart rate greater than the set HIGH heart rate limit.

The TACHY limit matches the high heart rate limit that is set under the Parameter Limit alarms. If the high heart rate limit is changed, the TACHY limit changes.

VT $>2$ Ventricular tachycardia $>2$ occurs when a run of ventricular beats is detected with a duration of less than six beats but longer than two beats and with an average heart rate that is greater or equal to 100 beats per minute. 


\section{Appendix B - Expressions of Alarm Frequency}

\section{Alarms per Patient per Day}

In ideal circumstances, alarm frequencies should be expressed in alarms per patient per day (APD). When calculated using the number of patient days for the data collection period, this not only accounts for unit size, but the patient load on the caregivers, presenting a more accurate quantification of the alarms occurring. It is important to note, however, that over short periods of data collection (less than one calendar month), the number of patient days for a unit may not be feasible to obtain.

Calculating alarms per patient per day for a single unit:

$$
A P D=\frac{\text { Alarms }}{\text { Patient } \cdot \text { Day }}=\frac{\text { Total Alarms }}{\text { Patient Days for Data Collection Period }}=\frac{A}{D_{P}}
$$

Calculating alarms per patient per day for " $n$ " units:

$$
A P D=\frac{i_{i=1}^{n} A_{i}}{n_{i=1}^{n} D_{P_{i}}}
$$

\section{Alarms per Bed per Day}

The expression method chosen for this study, alarms per bed per day does not account for patient load, but does account for unit size in its calculation. The information needed to calculate this value is readily available, and usually static, as unit sizes do not regularly change. Because these numbers remain static, this calculation method is viable for any duration period of data collection, and can be adjusted to suit other time intervals (i.e. hours, months, etc.) as needed.

Calculating alarms per patient per day for a single unit:

$$
A B D=\frac{\text { Alarms }}{\text { Bed } \cdot \text { Day }}=\frac{(\text { Total Alarms })}{\text { No. of Beds in Unit }(\text { No. of Days of Unit Data Collection })}=\frac{A}{B \cdot D}
$$

Calculating alarms per patient per day for " $n$ " units:

$$
A B D=\frac{{ }_{i=1}^{n} \frac{A_{i}}{D_{i}}}{n_{i=1}^{n} B_{i}}
$$

\section{Raw Alarm Frequency}

Expressing alarm frequency in terms of the raw number of alarms is limited in usefulness, as it may restrict the applicability of the data, particularly when comparing different units. Raw alarm frequency does not explicitly account for unit size or the length of the data collection period, but this may not be a factor when comparing data from the same unit over data collection periods of equal time. 


\section{Appendix C - Alarms per Bed per Day Tracking}

The table below summarizes the alarm collection for the 12 tracked units over the course of the study. Included in the table are the specialties for each of the units, along with their size in beds.

\begin{tabular}{|c|c|c|c|c|c|c|c|c|c|c|}
\hline \multicolumn{11}{|c|}{ Intensive Care Units (ICUs) } \\
\hline \multirow[b]{2}{*}{ Unit } & \multirow[b]{2}{*}{ Description } & \multirow[b]{2}{*}{ Beds } & \multicolumn{8}{|c|}{ Alarms/Bed/Day } \\
\hline & & & $\begin{array}{l}\text { Mar } \\
2013\end{array}$ & $\begin{array}{c}\text { Apr } \\
2013\end{array}$ & $\begin{array}{l}\text { May } \\
2013\end{array}$ & $\begin{array}{c}\text { Jun } \\
2013\end{array}$ & $\begin{array}{c}\text { Jul } \\
2013\end{array}$ & $\begin{array}{l}\text { Aug } \\
2013 \\
\end{array}$ & $\begin{array}{c}\text { Sep } \\
2013\end{array}$ & 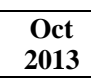 \\
\hline B7I & Surgical ICU, Trauma & 12 & 211.35 & 273.18 & 266.58 & 285.09 & 248.52 & 273.40 & 214.95 & 255.10 \\
\hline B9I & $\begin{array}{l}\text { Cardiothoracic Surgical } \\
\text { ICU }\end{array}$ & 16 & 272.54 & 303.64 & 392.55 & 358.21 & 321.93 & 405.88 & 403.53 & 417.23 \\
\hline B10I & Cardiac ICU & 12 & 242.26 & 430.25 & 283.39 & 311.18 & 254.85 & 319.65 & 270.17 & 314.94 \\
\hline B11I & Medical ICU & 16 & 273.00 & 295.85 & 290.51 & 289.90 & 266.24 & 256.45 & 318.20 & 367.55 \\
\hline C9I & Neurosurgical ICU & 18 & 217.41 & 269.60 & 306.52 & 292.77 & 235.92 & 257.22 & 291.18 & 241.41 \\
\hline \multicolumn{11}{|c|}{ Stepdown Units } \\
\hline & & & \multicolumn{8}{|c|}{ Alarms/Bed/Day } \\
\hline Unit & Description & Beds & $\begin{array}{l}\text { Mar } \\
2013\end{array}$ & $\begin{array}{l}\text { Apr } \\
2013\end{array}$ & $\begin{array}{l}\text { May } \\
2013\end{array}$ & $\begin{array}{c}\text { Jun } \\
2013\end{array}$ & $\begin{array}{c}\text { Jul } \\
2013\end{array}$ & $\begin{array}{l}\text { Aug } \\
2013\end{array}$ & $\begin{array}{c}\text { Sep } \\
2013\end{array}$ & $\begin{array}{c}\text { Oct } \\
2013\end{array}$ \\
\hline B7S & Surgical Stepdown & 3 & 203.85 & 376.31 & 539.11 & 360.30 & 387.29 & 469.84 & 297.63 & 352.37 \\
\hline B9S & $\begin{array}{l}\text { Cardiothoracic Surgical } \\
\text { Stepdown }\end{array}$ & 9 & 286.33 & 358.57 & 416.00 & 368.51 & 413.82 & 463.10 & 313.18 & 349.14 \\
\hline B11S & Medical Stepdown & 12 & 380.98 & 475.04 & 483.07 & 448.66 & 454.28 & 480.80 & 392.97 & 446.98 \\
\hline N9S & Neurosurgical Stepdown & 6 & 142.29 & 174.80 & 157.55 & 136.60 & 198.48 & 221.44 & 178.52 & 240.66 \\
\hline \multicolumn{11}{|c|}{ Medical/Surgical (Med/Surg) Units } \\
\hline & & & \multicolumn{8}{|c|}{ Alarms/Bed/Day } \\
\hline Unit & Description & Beds & $\begin{array}{l}\text { Mar } \\
2013\end{array}$ & $\begin{array}{l}\text { Apr } \\
2013\end{array}$ & $\begin{array}{l}\text { May } \\
2013\end{array}$ & $\begin{array}{c}\text { Jun } \\
2013\end{array}$ & $\begin{array}{c}\text { Jul } \\
2013 \\
\end{array}$ & $\begin{array}{l}\text { Aug } \\
2013 \\
\end{array}$ & $\begin{array}{c}\text { Sep } \\
2013\end{array}$ & $\begin{array}{l}\text { Oct } \\
2013\end{array}$ \\
\hline B10E & Cardiology & 14 & 140.85 & 161.74 & 152.86 & 139.22 & 193.77 & 214.97 & 185.25 & 175.43 \\
\hline B11E & Pulmonary/Medicine & 12 & 145.44 & 98.10 & 107.40 & 123.29 & 75.96 & 94.78 & 86.78 & 80.62 \\
\hline $\mathrm{C} 10$ & Cardiology & 26 & 151.12 & 152.43 & 141.51 & 142.84 & 121.56 & 122.54 & 209.95 & 117.34 \\
\hline
\end{tabular}

\begin{tabular}{|c|c|c|c|c|c|c|c|}
\hline \multicolumn{9}{|c|}{ Ranking of Units by Alarms per Bed per Day (A/B/D) } \\
\hline & Unit & A/B/D & \% Total & & Unit & A/B/D & \% Total \\
\hline $\mathbf{1}$ & B11S & 445.43 & $11.56 \%$ & $\mathbf{7}$ & C9I & 263.75 & $6.84 \%$ \\
\hline $\mathbf{2}$ & B7S & 373.69 & $9.70 \%$ & $\mathbf{8}$ & B7I & 253.47 & $6.58 \%$ \\
\hline $\mathbf{3}$ & B9S & 371.38 & $9.64 \%$ & $\mathbf{9}$ & N9S & 181.51 & $4.71 \%$ \\
\hline $\mathbf{4}$ & B9I & 359.49 & $9.33 \%$ & $\mathbf{1 0}$ & B10E & 170.61 & $4.43 \%$ \\
\hline $\mathbf{5}$ & B10I & 302.92 & $7.86 \%$ & $\mathbf{1 1}$ & C10 & 144.62 & $3.75 \%$ \\
\hline $\mathbf{6}$ & B11I & 294.63 & $7.65 \%$ & $\mathbf{1 2}$ & B11E & 101.53 & $2.63 \%$ \\
\hline
\end{tabular}




\section{Appendix D - Detailed Acuity Level Alarm Frequency Distributions}

\section{Alarm Frequency Distribution for Med/Surg Telemetry Units}

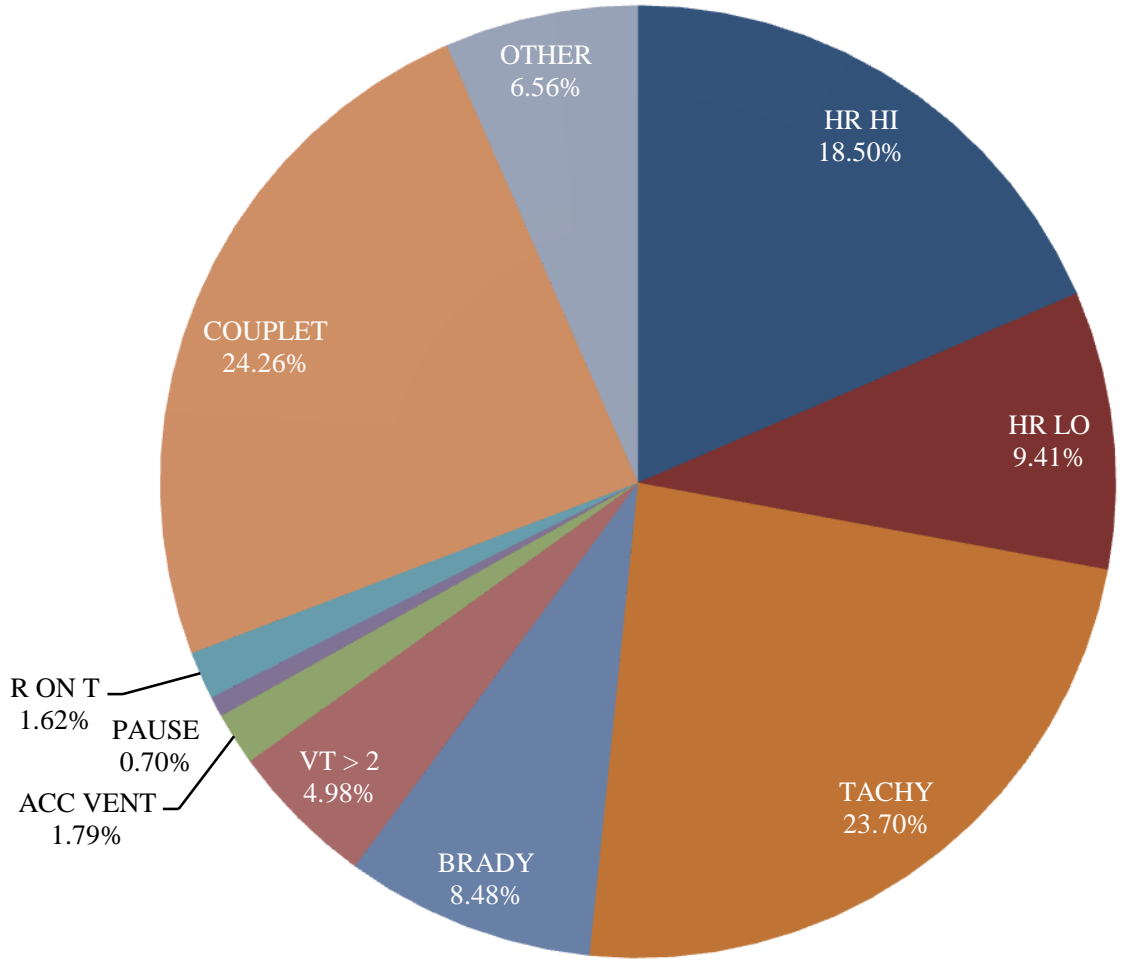

\begin{tabular}{|c|c|c|c|c|c|}
\hline Alarm Name & $\begin{array}{c}\text { B10E } \\
(\mathrm{A} / \mathrm{B} / \mathrm{D})\end{array}$ & $\begin{array}{c}\text { B11E } \\
(\mathrm{A} / \mathrm{B} / \mathrm{D})\end{array}$ & $\begin{array}{c}\text { C10 } \\
(\mathrm{A} / \mathrm{B} / \mathrm{D})\end{array}$ & $\begin{array}{c}\text { Total } \\
\text { (A/B/D) }\end{array}$ & $\begin{array}{l}\% \text { Total } \\
\text { Alarms }\end{array}$ \\
\hline HR HI & 31.31 & 25.83 & 23.65 & 26.21 & $18.50 \%$ \\
\hline HR LO & 14.52 & 7.49 & 15.40 & 13.34 & $9.41 \%$ \\
\hline SPO2 LO & 0.00 & 0.00 & 0.00 & 0.00 & $0.00 \%$ \\
\hline NBP S HI & 0.00 & 0.00 & 0.00 & 0.00 & $0.00 \%$ \\
\hline NBP S LO & 0.00 & 0.00 & 0.00 & 0.00 & $0.00 \%$ \\
\hline NBP D HI & 0.00 & 0.00 & 0.00 & 0.00 & $0.00 \%$ \\
\hline NBP D LO & 0.00 & 0.00 & 0.00 & 0.00 & $0.00 \%$ \\
\hline NBP M HI & 0.00 & 0.00 & 0.00 & 0.00 & $0.00 \%$ \\
\hline NBP M LO & 0.00 & 0.00 & 0.00 & 0.00 & $0.00 \%$ \\
\hline RSP HI & 0.00 & 0.00 & 0.00 & 0.00 & $0.00 \%$ \\
\hline RSP LO & 0.00 & 0.00 & 0.00 & 0.00 & $0.00 \%$ \\
\hline TACHY & 38.91 & 29.97 & 32.38 & 33.58 & $23.70 \%$ \\
\hline BRADY & 13.53 & 5.65 & 14.13 & 12.01 & $8.48 \%$ \\
\hline VT > 2 & 8.89 & 3.04 & 7.93 & 7.06 & $4.98 \%$ \\
\hline ACC VENT & 3.36 & 1.01 & 2.80 & 2.54 & $1.79 \%$ \\
\hline PAUSE & 1.04 & 0.76 & 1.07 & 0.99 & $0.70 \%$ \\
\hline R ON T & 2.51 & 1.70 & 2.45 & 2.29 & $1.62 \%$ \\
\hline COUPLET & 45.17 & 18.70 & 35.78 & 34.36 & $24.26 \%$ \\
\hline OTHER & 11.38 & 7.38 & 9.05 & 9.29 & $6.56 \%$ \\
\hline Total & 170.61 & 101.53 & 144.62 & 141.68 & $100.00 \%$ \\
\hline
\end{tabular}

Note: The grayed out alarms refer to alarms that were tracked in the study, but not used with the monitoring devices at this acuity level. 


\section{Alarm Frequency Distribution for Stepdown Units}

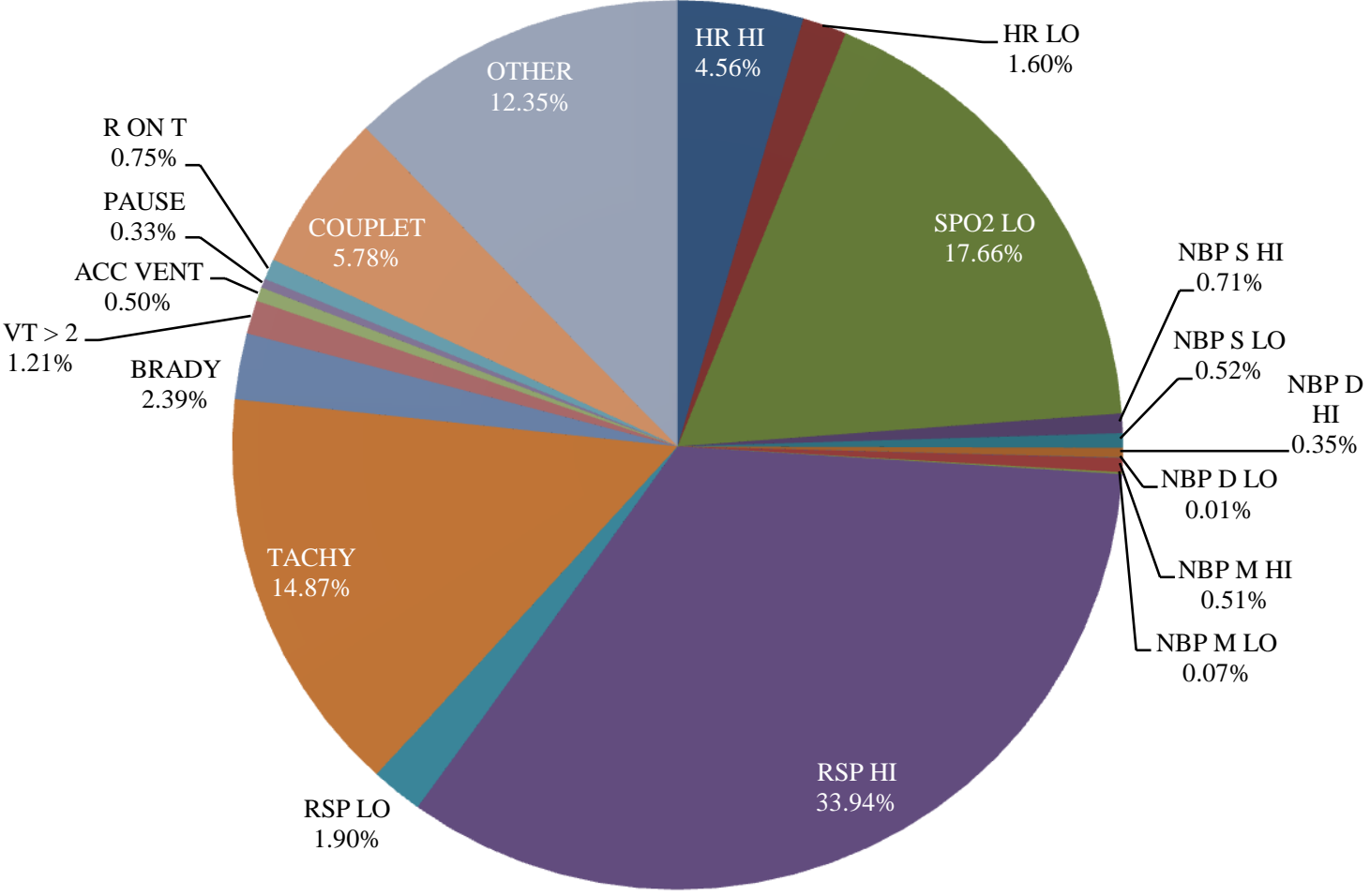

\begin{tabular}{|c|c|c|c|c|c|c|}
$\begin{array}{c}\text { Alarm } \\
\text { Name }\end{array}$ & $\begin{array}{c}\text { B7S } \\
(\mathbf{A} / \mathbf{B} / \mathbf{D})\end{array}$ & $\begin{array}{c}\text { B9S } \\
(\mathbf{A} / \mathbf{B} / \mathbf{D})\end{array}$ & \multicolumn{1}{c}{$\begin{array}{c}\text { B11S } \\
(\mathbf{A} / \mathbf{B} / \mathbf{D})\end{array}$} & $\begin{array}{c}\text { N9S } \\
(\mathbf{A} / \mathbf{B} / \mathbf{D})\end{array}$ & $\begin{array}{c}\text { Average } \\
(\mathbf{A} / \mathbf{B} / \mathbf{D})\end{array}$ & $\begin{array}{c}\text { \% Total } \\
\text { Alarms }\end{array}$ \\
\hline HR HI & 13.94 & 11.34 & 21.84 & 13.46 & 16.72 & $4.56 \%$ \\
\hline HR LO & 4.75 & 4.45 & 6.30 & 7.72 & 5.87 & $1.60 \%$ \\
\hline SPO2 LO & 47.77 & 63.73 & 81.25 & 42.07 & 64.81 & $17.66 \%$ \\
\hline NBP S HI & 2.26 & 1.46 & 3.09 & 3.48 & 2.60 & $0.71 \%$ \\
\hline NBP S LO & 1.34 & 1.90 & 2.75 & 0.62 & 1.93 & $0.52 \%$ \\
\hline NBP D HI & 0.89 & 0.66 & 1.85 & 1.33 & 1.29 & $0.35 \%$ \\
\hline NBP D LO & 0.05 & 0.05 & 0.08 & 0.01 & 0.05 & $0.01 \%$ \\
\hline NBP M HI & 1.44 & 1.65 & 2.14 & 1.85 & 1.87 & $0.51 \%$ \\
\hline NBP M LO & 0.10 & 0.19 & 0.44 & 0.00 & 0.24 & $0.07 \%$ \\
\hline RSP HI & 156.38 & 128.67 & 168.75 & 13.93 & 124.53 & $33.94 \%$ \\
\hline RSP LO & 15.21 & 8.22 & 6.35 & 2.16 & 6.96 & $1.90 \%$ \\
\hline TACHY & 50.10 & 47.18 & 68.19 & 40.62 & 54.56 & $14.87 \%$ \\
\hline BRADY & 7.06 & 8.46 & 8.81 & 9.98 & 8.76 & $2.39 \%$ \\
\hline VT > 2 & 1.62 & 7.61 & 3.77 & 2.50 & 4.45 & $1.21 \%$ \\
\hline ACC VENT & 0.10 & 4.66 & 0.57 & 1.00 & 1.83 & $0.50 \%$ \\
\hline PAUSE & 0.26 & 2.28 & 0.86 & 0.80 & 1.21 & $0.33 \%$ \\
\hline R ON T & 1.47 & 5.07 & 2.06 & 1.30 & 2.75 & $0.75 \%$ \\
\hline COUPLET & 12.07 & 34.06 & 16.56 & 15.77 & 21.20 & $5.78 \%$ \\
\hline OTHER & 56.86 & 50.45 & 49.77 & 22.93 & 45.31 & $12.35 \%$ \\
\hline Total & 373.69 & 382.06 & 445.43 & 181.51 & 366.95 & $100.00 \%$ \\
\hline
\end{tabular}




\section{Alarm Frequency Distribution for ICUs}

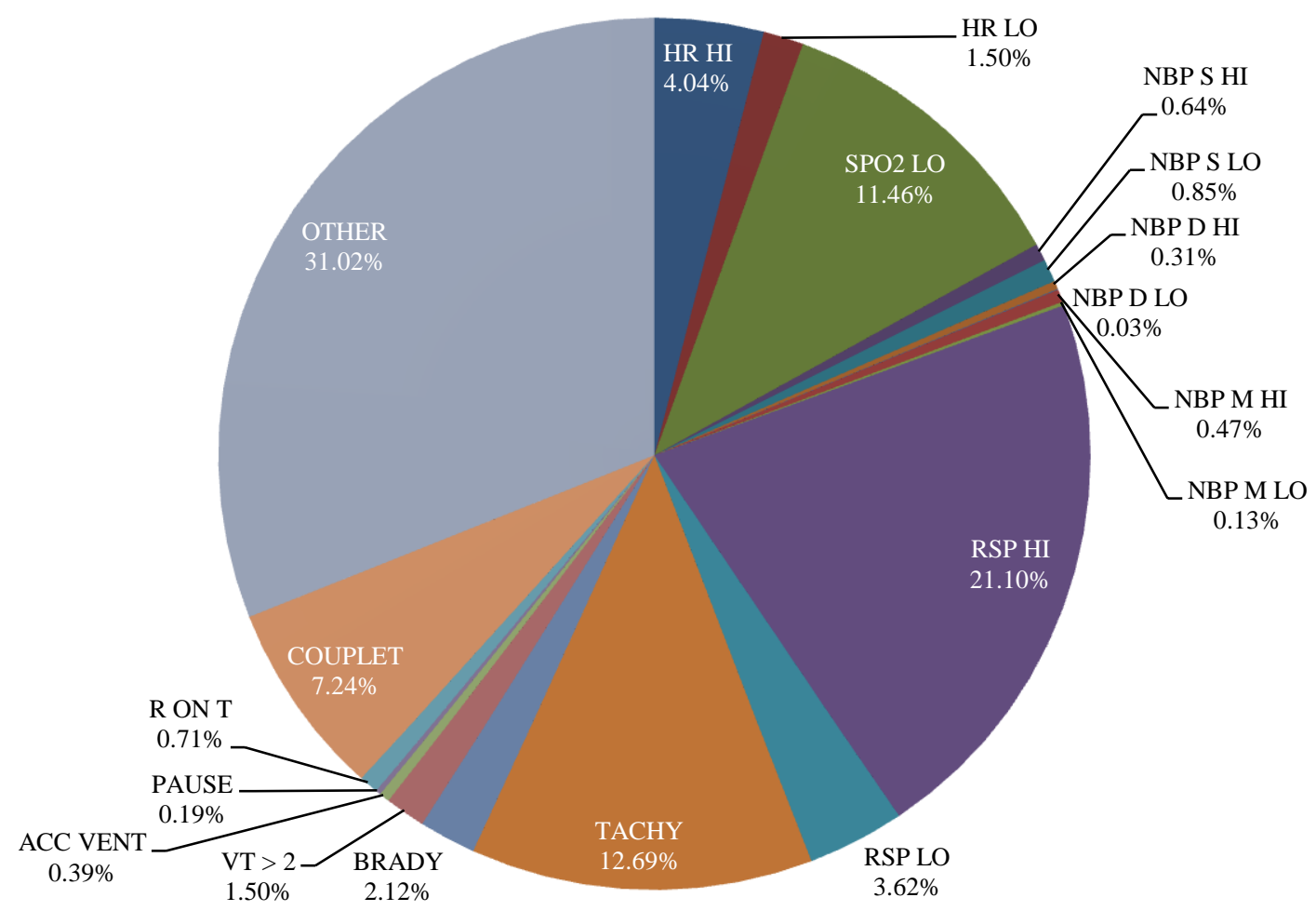

\begin{tabular}{c|c|c|c|c|c|c|c|}
$\begin{array}{c}\text { Alarm } \\
\text { Name }\end{array}$ & $\begin{array}{c}\text { B7I } \\
(\mathbf{A} / \mathbf{B} / \mathbf{D})\end{array}$ & $\begin{array}{c}\text { B9I } \\
(\mathbf{A} / \mathbf{B} / \mathbf{D})\end{array}$ & $\begin{array}{c}\text { B10I } \\
(\mathbf{A} / \mathbf{B} / \mathbf{D})\end{array}$ & $\begin{array}{c}\text { B11I } \\
(\mathbf{A} / \mathbf{B} / \mathbf{D})\end{array}$ & $\begin{array}{c}\text { C9I } \\
(\mathbf{A} / \mathbf{B} / \mathbf{D})\end{array}$ & $\begin{array}{c}\text { Total } \\
(\mathbf{A} / \mathbf{B} / \mathbf{D})\end{array}$ & $\begin{array}{c}\text { \% Total } \\
\text { Alarms }\end{array}$ \\
\hline HR HI & 7.45 & 13.87 & 6.47 & 15.42 & 13.78 & 11.94 & $4.04 \%$ \\
\hline HR LO & 1.74 & 2.77 & 4.52 & 4.60 & 7.48 & 4.43 & $1.50 \%$ \\
\hline SPO2 LO & 32.60 & 35.68 & 40.60 & 47.80 & 16.41 & 33.91 & $11.46 \%$ \\
\hline NBP S HI & 1.72 & 1.01 & 1.41 & 2.68 & 2.42 & 1.90 & $0.64 \%$ \\
\hline NBP S LO & 1.96 & 2.07 & 3.33 & 4.16 & 1.23 & 2.50 & $0.85 \%$ \\
\hline NBP D HI & 0.77 & 0.38 & 0.92 & 1.55 & 0.88 & 0.90 & $0.31 \%$ \\
\hline NBP D LO & 0.08 & 0.06 & 0.11 & 0.14 & 0.06 & 0.09 & $0.03 \%$ \\
\hline NBP M HI & 1.15 & 1.15 & 1.28 & 2.26 & 1.07 & 1.39 & $0.47 \%$ \\
\hline NBP M LO & 0.29 & 0.27 & 0.57 & 0.62 & 0.26 & 0.39 & $0.13 \%$ \\
\hline RSP HI & 57.39 & 58.79 & 79.89 & 76.71 & 44.62 & 62.41 & $21.10 \%$ \\
\hline RSP LO & 13.66 & 10.91 & 11.35 & 8.01 & 10.49 & 10.70 & $3.62 \%$ \\
\hline TACHY & 20.08 & 48.85 & 27.79 & 49.30 & 35.13 & 37.53 & $12.69 \%$ \\
\hline BRADY & 2.87 & 4.62 & 6.63 & 6.57 & 9.45 & 6.26 & $2.12 \%$ \\
\hline VT > 2 & 2.23 & 6.51 & 9.30 & 3.81 & 1.42 & 4.45 & $1.50 \%$ \\
\hline ACC VENT & 0.34 & 2.41 & 1.46 & 0.83 & 0.64 & 1.15 & $0.39 \%$ \\
\hline PAUSE & 0.21 & 0.93 & 1.01 & 0.59 & 0.15 & 0.56 & $0.19 \%$ \\
\hline R ON T & 0.83 & 4.11 & 1.90 & 1.24 & 2.10 & 2.11 & $0.71 \%$ \\
\hline COUPLET & 17.71 & 28.93 & 37.67 & 17.59 & 9.82 & 21.43 & $7.24 \%$ \\
\hline OTHER & 90.41 & 136.17 & 66.71 & 50.77 & 106.38 & 91.77 & $31.02 \%$ \\
\hline Total & 253.47 & 359.49 & 302.92 & 294.63 & 263.75 & 295.81 & $100.00 \%$ \\
\hline
\end{tabular}




\section{Appendix E - Detailed Alarm Condition Distributions}

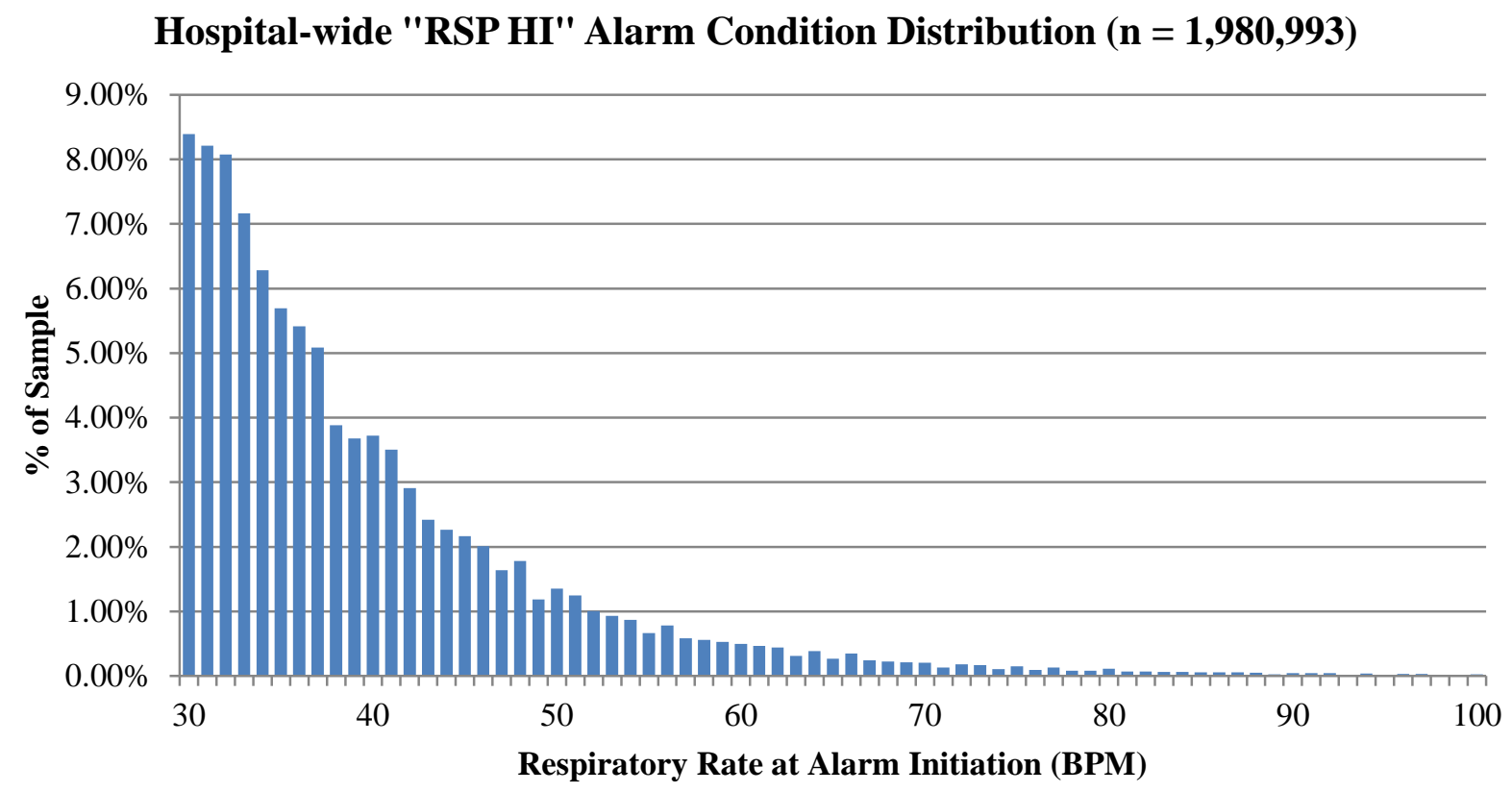

\begin{tabular}{c|c|c|c|c|c|}
$\begin{array}{c}\text { Resp Rate } \\
\text { (BPM) }\end{array}$ & \% Total & $\begin{array}{c}\text { Resp Rate } \\
\text { (BPM) }\end{array}$ & \% Total & $\begin{array}{c}\text { Resp Rate } \\
\text { (BPM) }\end{array}$ & \% Total \\
\hline 30 & $8.39 \%$ & 54 & $0.87 \%$ & 78 & $0.08 \%$ \\
\hline 31 & $8.21 \%$ & 55 & $0.66 \%$ & 79 & $0.08 \%$ \\
\hline 32 & $8.08 \%$ & 56 & $0.78 \%$ & 80 & $0.11 \%$ \\
\hline 33 & $7.16 \%$ & 57 & $0.58 \%$ & 81 & $0.07 \%$ \\
\hline 34 & $6.28 \%$ & 58 & $0.56 \%$ & 82 & $0.07 \%$ \\
\hline 35 & $5.70 \%$ & 59 & $0.53 \%$ & 83 & $0.07 \%$ \\
\hline 36 & $5.42 \%$ & 60 & $0.50 \%$ & 84 & $0.06 \%$ \\
\hline 37 & $5.09 \%$ & 61 & $0.46 \%$ & 85 & $0.06 \%$ \\
\hline 38 & $3.88 \%$ & 62 & $0.44 \%$ & 86 & $0.06 \%$ \\
\hline 39 & $3.68 \%$ & 63 & $0.31 \%$ & 87 & $0.06 \%$ \\
\hline 40 & $3.72 \%$ & 64 & $0.39 \%$ & 88 & $0.05 \%$ \\
\hline 41 & $3.51 \%$ & 65 & $0.27 \%$ & 89 & $0.02 \%$ \\
\hline 42 & $2.91 \%$ & 66 & $0.35 \%$ & 90 & $0.04 \%$ \\
\hline 43 & $2.42 \%$ & 67 & $0.24 \%$ & 91 & $0.04 \%$ \\
\hline 44 & $2.27 \%$ & 68 & $0.22 \%$ & 92 & $0.04 \%$ \\
\hline 45 & $2.17 \%$ & 69 & $0.21 \%$ & 93 & $0.02 \%$ \\
\hline 46 & $2.01 \%$ & 70 & $0.21 \%$ & 94 & $0.04 \%$ \\
\hline 47 & $1.64 \%$ & 71 & $0.13 \%$ & 95 & $0.02 \%$ \\
\hline 48 & $1.78 \%$ & 72 & $0.18 \%$ & 96 & $0.03 \%$ \\
\hline 49 & $1.18 \%$ & 73 & $0.17 \%$ & 97 & $0.03 \%$ \\
\hline 50 & $1.35 \%$ & 74 & $0.11 \%$ & 98 & $0.01 \%$ \\
\hline 51 & $1.25 \%$ & 75 & $0.15 \%$ & 99 & $0.02 \%$ \\
\hline 52 & $1.00 \%$ & 76 & $0.10 \%$ & 100 & $0.03 \%$ \\
\hline 53 & $0.93 \%$ & 77 & $0.13 \%$ & & \\
\hline & & & & & \\
\hline
\end{tabular}




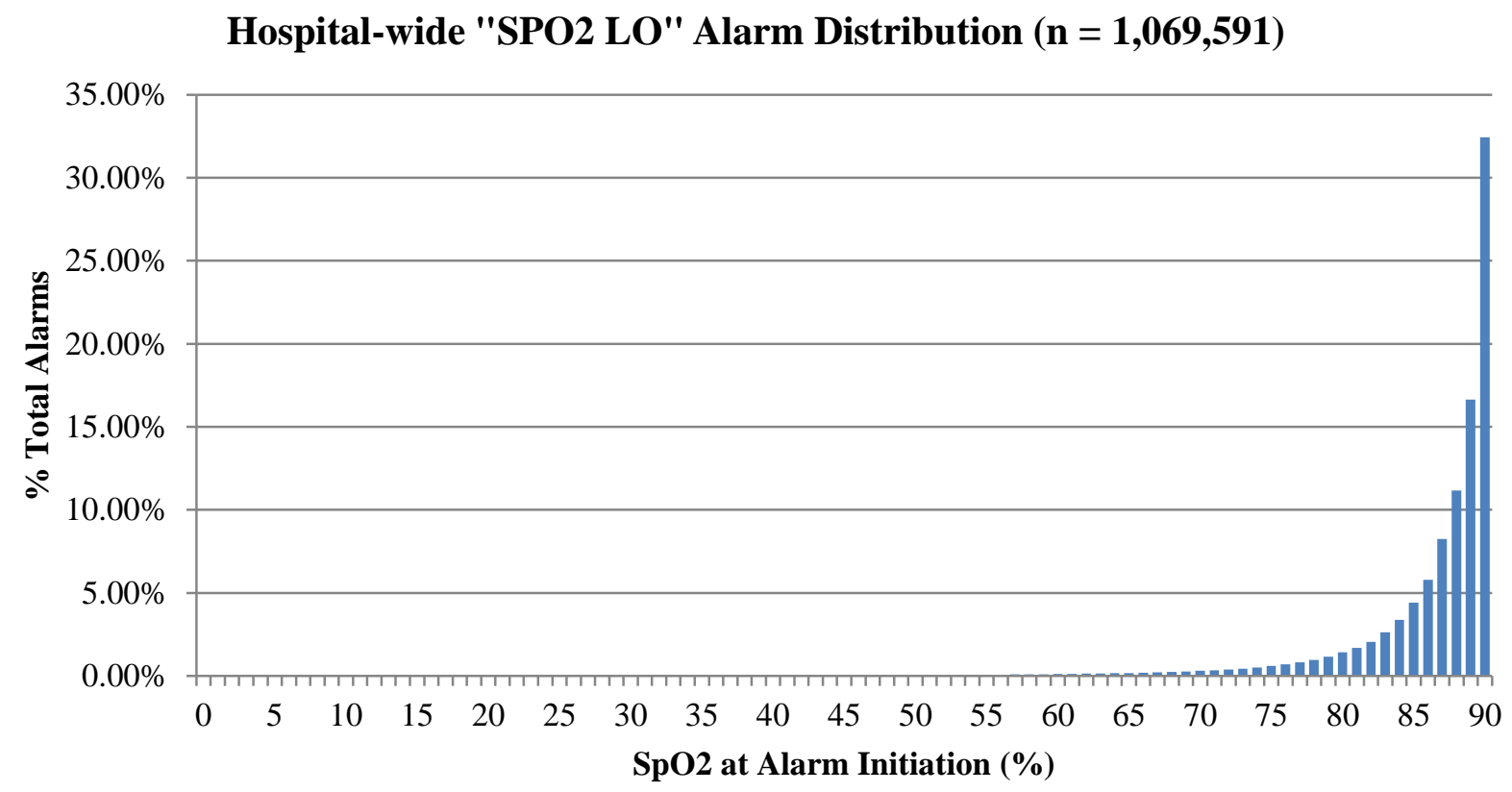

\begin{tabular}{|c|c|c|c|c|c|c|c|}
\hline $\mathbf{S p O}_{\mathbf{2}}(\mathbf{\%})$ & $\mathbf{\%} \mathbf{T o t a l}$ & $\mathbf{S p O}_{\mathbf{2}}(\mathbf{\%})$ & $\mathbf{\%} \mathbf{T o t a l}$ & $\mathbf{S p O}_{\mathbf{2}}(\mathbf{\%})$ & $\mathbf{\%} \mathbf{T o t a l}$ & $\mathbf{S p O}_{\mathbf{2}}(\boldsymbol{\%})$ & $\mathbf{\%} \mathbf{T o t a l}$ \\
\hline 0 & $0.00 \%$ & 23 & $0.01 \%$ & 46 & $0.08 \%$ & 69 & $0.27 \%$ \\
\hline 1 & $0.01 \%$ & 24 & $0.02 \%$ & 47 & $0.07 \%$ & 70 & $0.32 \%$ \\
\hline 2 & $0.01 \%$ & 25 & $0.02 \%$ & 48 & $0.06 \%$ & 71 & $0.34 \%$ \\
\hline 3 & $0.01 \%$ & 26 & $0.02 \%$ & 49 & $0.07 \%$ & 72 & $0.39 \%$ \\
\hline 4 & $0.01 \%$ & 27 & $0.02 \%$ & 50 & $0.08 \%$ & 73 & $0.44 \%$ \\
\hline 5 & $0.01 \%$ & 28 & $0.02 \%$ & 51 & $0.06 \%$ & 74 & $0.51 \%$ \\
\hline 6 & $0.01 \%$ & 29 & $0.02 \%$ & 52 & $0.07 \%$ & 75 & $0.60 \%$ \\
\hline 7 & $0.01 \%$ & 30 & $0.02 \%$ & 53 & $0.07 \%$ & 76 & $0.70 \%$ \\
\hline 8 & $0.01 \%$ & 31 & $0.02 \%$ & 54 & $0.07 \%$ & 77 & $0.82 \%$ \\
\hline 9 & $0.01 \%$ & 32 & $0.02 \%$ & 55 & $0.08 \%$ & 78 & $0.96 \%$ \\
\hline 10 & $0.01 \%$ & 33 & $0.02 \%$ & 56 & $0.09 \%$ & 79 & $1.15 \%$ \\
\hline 11 & $0.01 \%$ & 34 & $0.03 \%$ & 57 & $0.09 \%$ & 80 & $1.42 \%$ \\
\hline 12 & $0.01 \%$ & 35 & $0.03 \%$ & 58 & $0.10 \%$ & 81 & $1.69 \%$ \\
\hline 13 & $0.01 \%$ & 36 & $0.03 \%$ & 59 & $0.11 \%$ & 82 & $2.06 \%$ \\
\hline 14 & $0.01 \%$ & 37 & $0.03 \%$ & 60 & $0.12 \%$ & 83 & $2.63 \%$ \\
\hline 15 & $0.01 \%$ & 38 & $0.03 \%$ & 61 & $0.13 \%$ & 84 & $3.39 \%$ \\
\hline 16 & $0.01 \%$ & 39 & $0.04 \%$ & 62 & $0.14 \%$ & 85 & $4.41 \%$ \\
\hline 17 & $0.01 \%$ & 40 & $0.04 \%$ & 63 & $0.15 \%$ & 86 & $5.79 \%$ \\
\hline 18 & $0.01 \%$ & 41 & $0.04 \%$ & 64 & $0.17 \%$ & 87 & $8.25 \%$ \\
\hline 19 & $0.01 \%$ & 42 & $0.04 \%$ & 65 & $0.18 \%$ & 88 & $11.17 \%$ \\
\hline 20 & $0.01 \%$ & 43 & $0.05 \%$ & 66 & $0.20 \%$ & 89 & $16.64 \%$ \\
\hline 21 & $0.01 \%$ & 44 & $0.06 \%$ & 67 & $0.22 \%$ & 90 & $32.43 \%$ \\
\hline 22 & $0.01 \%$ & 45 & $0.07 \%$ & 68 & $0.24 \%$ & & \\
\hline
\end{tabular}


Hospital-wide "HR HI" Alarm Distribution $(n=673,450)$

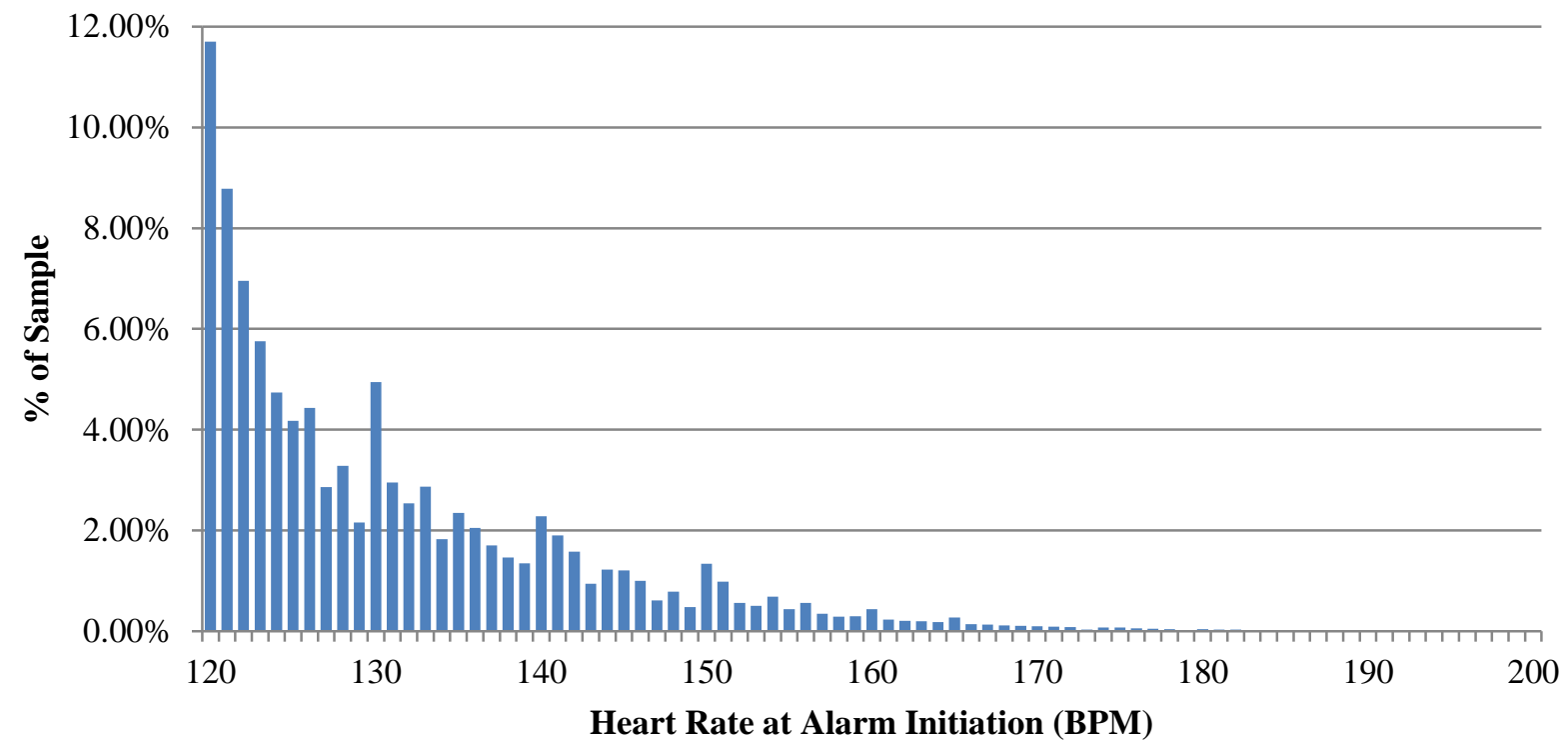

\begin{tabular}{|c|c|c|c|c|c|c|c|}
$\begin{array}{c}\text { Heart } \\
\text { Rate } \\
(\mathbf{B P M})\end{array}$ & \% Total & $\begin{array}{c}\text { Heart } \\
\text { Rate } \\
\text { (BPM) }\end{array}$ & \% Total & $\begin{array}{c}\text { Heart } \\
\text { Rate } \\
\text { (BPM) }\end{array}$ & \% Total & $\begin{array}{c}\text { Heart } \\
\text { Rate } \\
(\mathbf{B P M})\end{array}$ & \% Total \\
\hline 120 & $11.70 \%$ & 141 & $1.90 \%$ & 161 & $0.23 \%$ & 181 & $0.04 \%$ \\
\hline 121 & $8.78 \%$ & 142 & $1.58 \%$ & 162 & $0.21 \%$ & 182 & $0.03 \%$ \\
\hline 122 & $6.95 \%$ & 143 & $0.94 \%$ & 163 & $0.20 \%$ & 183 & $0.02 \%$ \\
\hline 123 & $5.75 \%$ & 144 & $1.22 \%$ & 164 & $0.18 \%$ & 184 & $0.03 \%$ \\
\hline 124 & $4.74 \%$ & 145 & $1.21 \%$ & 165 & $0.27 \%$ & 185 & $0.03 \%$ \\
\hline 125 & $4.18 \%$ & 146 & $1.00 \%$ & 166 & $0.14 \%$ & 186 & $0.01 \%$ \\
\hline 126 & $4.43 \%$ & 147 & $0.61 \%$ & 167 & $0.14 \%$ & 187 & $0.02 \%$ \\
\hline 127 & $2.86 \%$ & 148 & $0.79 \%$ & 168 & $0.12 \%$ & 188 & $0.02 \%$ \\
\hline 128 & $3.29 \%$ & 149 & $0.48 \%$ & 169 & $0.11 \%$ & 189 & $0.01 \%$ \\
\hline 129 & $2.16 \%$ & 150 & $1.34 \%$ & 170 & $0.10 \%$ & 190 & $0.02 \%$ \\
\hline 130 & $4.94 \%$ & 151 & $0.98 \%$ & 171 & $0.09 \%$ & 191 & $0.01 \%$ \\
\hline 131 & $2.96 \%$ & 152 & $0.56 \%$ & 172 & $0.08 \%$ & 192 & $0.02 \%$ \\
\hline 132 & $2.54 \%$ & 153 & $0.51 \%$ & 173 & $0.04 \%$ & 193 & $0.01 \%$ \\
\hline 133 & $2.87 \%$ & 154 & $0.68 \%$ & 174 & $0.07 \%$ & 194 & $0.01 \%$ \\
\hline 134 & $1.82 \%$ & 155 & $0.44 \%$ & 175 & $0.08 \%$ & 195 & $0.01 \%$ \\
\hline 135 & $2.35 \%$ & 156 & $0.57 \%$ & 176 & $0.06 \%$ & 196 & $0.01 \%$ \\
\hline 136 & $2.05 \%$ & 157 & $0.35 \%$ & 177 & $0.05 \%$ & 197 & $0.01 \%$ \\
\hline 137 & $1.70 \%$ & 158 & $0.29 \%$ & 178 & $0.05 \%$ & 198 & $0.01 \%$ \\
\hline 138 & $1.47 \%$ & 159 & $0.30 \%$ & 179 & $0.02 \%$ & 199 & $0.01 \%$ \\
\hline 139 & $1.35 \%$ & 160 & $0.44 \%$ & 180 & $0.05 \%$ & 200 & $0.01 \%$ \\
\hline 140 & $2.28 \%$ & & & & & & \\
\hline
\end{tabular}




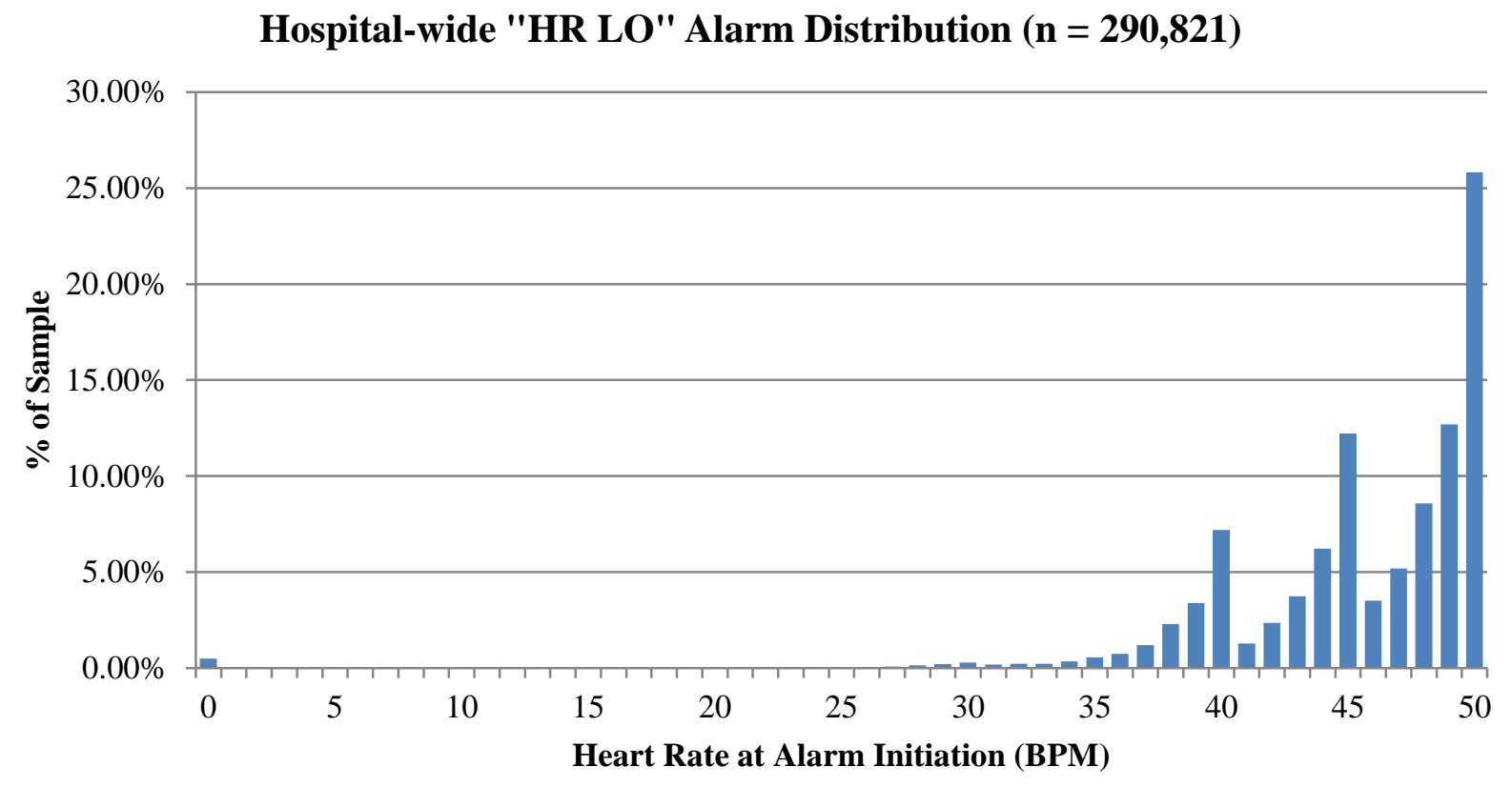

\begin{tabular}{|c|c|c|c|c|c|}
$\begin{array}{c}\text { Heart Rate } \\
(\mathbf{B P M})\end{array}$ & \% Total & $\begin{array}{c}\text { Heart Rate } \\
(\mathbf{B P M})\end{array}$ & $\%$ Total & $\begin{array}{c}\text { Heart Rate } \\
\text { (BPM) }\end{array}$ & \% Total \\
\hline 0 & $0.50 \%$ & 17 & $0.03 \%$ & 34 & $0.35 \%$ \\
\hline 1 & $0.02 \%$ & 18 & $0.03 \%$ & 35 & $0.55 \%$ \\
\hline 2 & $0.02 \%$ & 19 & $0.03 \%$ & 36 & $0.74 \%$ \\
\hline 3 & $0.02 \%$ & 20 & $0.03 \%$ & 37 & $1.21 \%$ \\
\hline 4 & $0.02 \%$ & 21 & $0.03 \%$ & 38 & $2.30 \%$ \\
\hline 5 & $0.02 \%$ & 22 & $0.03 \%$ & 39 & $3.40 \%$ \\
\hline 6 & $0.02 \%$ & 23 & $0.03 \%$ & 40 & $7.20 \%$ \\
\hline 7 & $0.02 \%$ & 24 & $0.04 \%$ & 41 & $1.28 \%$ \\
\hline 8 & $0.03 \%$ & 25 & $0.06 \%$ & 42 & $2.36 \%$ \\
\hline 9 & $0.02 \%$ & 26 & $0.06 \%$ & 43 & $3.74 \%$ \\
\hline 10 & $0.02 \%$ & 27 & $0.09 \%$ & 44 & $6.22 \%$ \\
\hline 11 & $0.02 \%$ & 28 & $0.14 \%$ & 45 & $12.22 \%$ \\
\hline 12 & $0.02 \%$ & 29 & $0.22 \%$ & 46 & $3.51 \%$ \\
\hline 13 & $0.02 \%$ & 30 & $0.29 \%$ & 47 & $5.20 \%$ \\
\hline 14 & $0.03 \%$ & 31 & $0.19 \%$ & 48 & $8.58 \%$ \\
\hline 15 & $0.03 \%$ & 32 & $0.22 \%$ & 49 & $12.69 \%$ \\
\hline 16 & $0.03 \%$ & 33 & $0.22 \%$ & 50 & $25.82 \%$ \\
\hline
\end{tabular}




\section{Appendix F - Coefficients of Determination $\left(\mathbf{R}^{2}\right)$ for Select Distributions}

\section{Comparing "RSP HI" Alarm Distributions by Acuity Level}

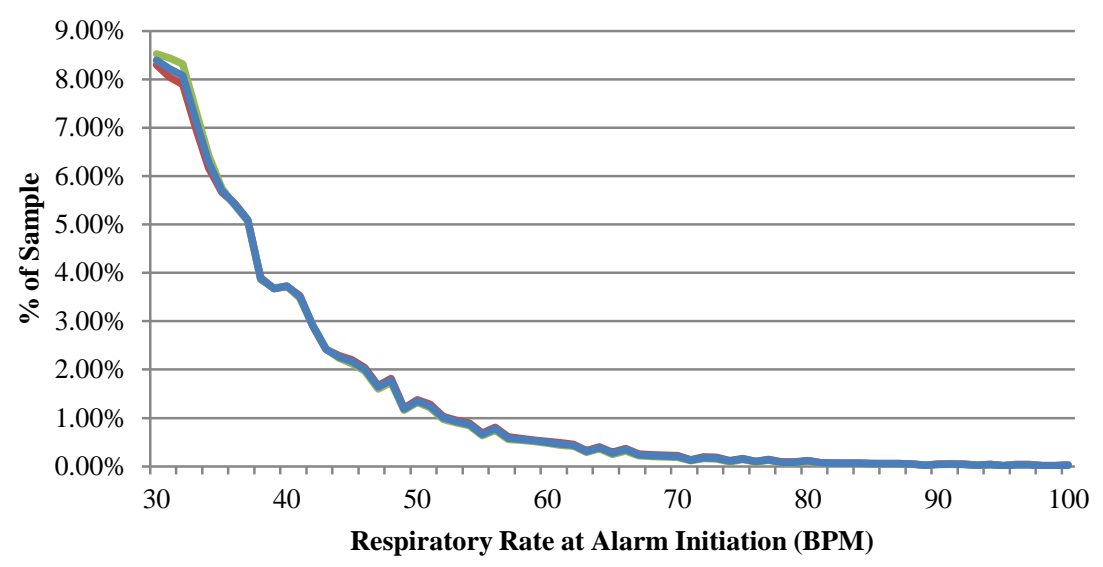

$\longrightarrow \mathrm{ICU} \longrightarrow$ Stepdown $\longrightarrow$ All Units

Comparing "SPO2 LO" Distributions by Acuity Level

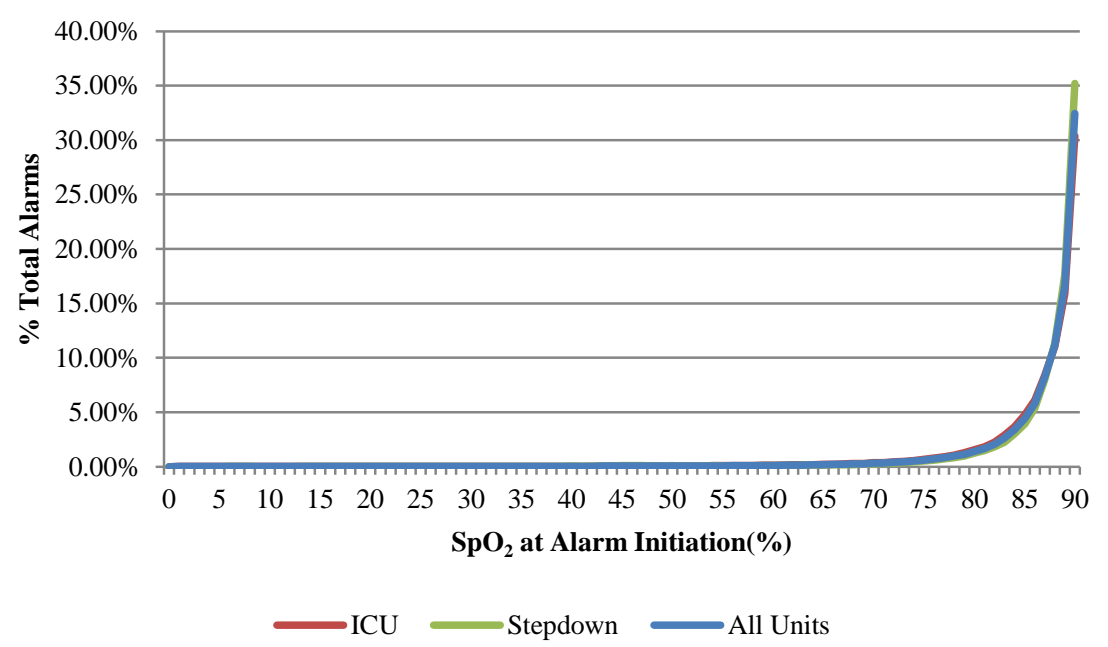

Comparison

Avg to B7I

Avg to B9I

Avg to B10I

Avg to B11I

Avg to C9I

Avg to B7S

Avg to B9S

Avg to B11S

Avg to N9S
$\mathbf{R}^{2}$

0.999

0.999

0.994

1.000

0.998

0.999

1.000

0.999

0.997

\begin{tabular}{|c|c|}
\hline Comparison & $\mathbf{R}^{\mathbf{2}}$ \\
\hline Avg to B7I & 0.989 \\
\hline Avg to B9I & 0.997 \\
\hline Avg to B10I & 0.946 \\
\hline Avg to B11I & 0.997 \\
\hline Avg to C9I & 0.996 \\
\hline Avg to B7S & 0.996 \\
\hline Avg to B9S & 0.986 \\
\hline Avg to B11S & 0.994 \\
\hline Avg to N9S & 0.996 \\
\hline
\end{tabular}


Comparing "HR HI" Alarm Distributions by Acuity Level

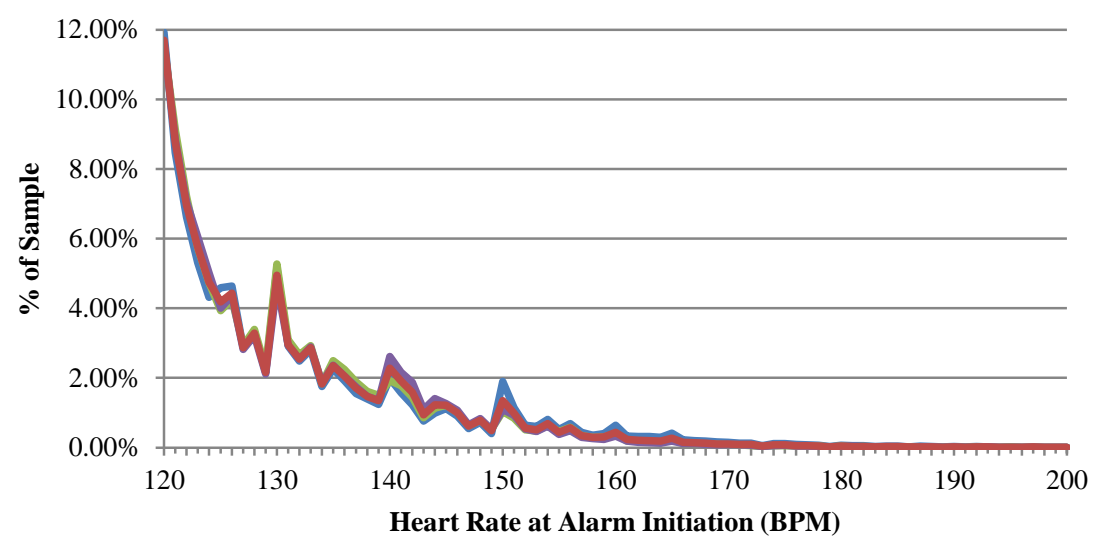

$\longrightarrow \mathrm{ICU} \longrightarrow$ Stepdown $\longrightarrow$ Med/Surg $\longrightarrow$ All Units

Comparing "HR LO" Alarm Distributions by Acuity Level

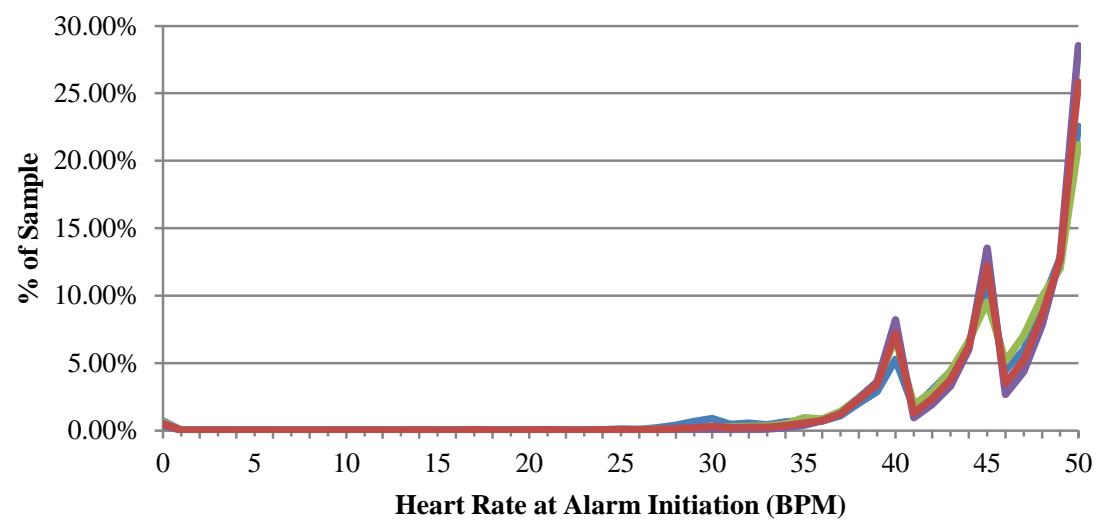

$\longrightarrow \mathrm{ICU} \longrightarrow$ Stepdown $\longrightarrow$ Med/Surg $\longrightarrow$ All Units
Comparison

$\mathbf{R}^{2}$

Avg to B7I

Avg to B9I

Avg to B10I

Avg to B11

Avg to C9I

Avg to B7S

Avg to B9S

Avg to B11S

Avg to N9S

Avg to B10E

Avg to B11E

Avg to $\mathrm{C} 10$
0.956

0.984

0.972

0.891

0.964

0.983

0.977

0.995

0.97

0.989

0.967

\begin{tabular}{|c|c|}
\hline Comparison & $\mathbf{R}^{\mathbf{2}}$ \\
\hline Avg to B7I & 0.889 \\
\hline Avg to B9I & 0.920 \\
\hline Avg to B10I & 0.935 \\
\hline Avg to B11I & 0.979 \\
\hline Avg to C9I & 0.988 \\
\hline Avg to B7S & 0.855 \\
\hline Avg to B9S & 0.902 \\
\hline Avg to B11S & 0.979 \\
\hline Avg to N9S & 0.981 \\
\hline Avg to B10E & 0.988 \\
\hline Avg to B11E & 0.935 \\
\hline Avg to C10 & 0.781 \\
\hline
\end{tabular}




\section{References}

[1] Chambrin MC, Ravaux P, Calvelo-Aros D, Jaborska A, Chopin C, Boniface B. "Multicentric study of monitoring alarms in the adult intensive care unit (ICU): a descriptive analysis." Intensive Care Med. 1999, 25, pp. 1360-1366.

[2] Brezmitz S. "Cry Wolf: The Psychology of False Alarms.” New Jersey: Lawrence Erlbaum, Hillsdale, 1984.

[3] Cvach M. "Monitor Alarm Fatigue: An Integrative Review." Biomedical Instrumentation \& Technology, July/August 2012, 46(4), pp. 268-277.

[4] The Joint Commission. "Medical device alarm safety in hospitals." Sentinel Event Alert, April 8, 2013, Issue 50.

[5] Kowalczyk L. “'Alarm Fatigue' a factor in 2nd death.” The Boston Globe, Sep. 21, 2011.<http://www.boston.com/lifestyle/health/articles/2011/09/21/umass_hospital_has_s econd_death_involving_alarm_fatigue/?page=full>.

[6] Kowalczyk L. "'Alarm Fatigue' linked to patient's death.” The Boston Globe, Apr. 3, 2010. <http://www.boston.com/news/local/massachusetts/articles/2010/04/03/ alarm_fatigue_linked_to_heart_patients_death_at_mass_general/>.

[7] Taenzer A et al. "Impact of pulse oximetry surveillance on rescue events and intensive care unit transfers: A before-and-after concurrence study." Anesthesiology 2010; 112:282-7.

[8] ECRI Institute. “Top 10 Health Technology Hazards for 2013.” Health Devices. 2012; 41(11).

[9] The Joint Commission. "National Patient Safety Goals Effective January 1, 2014." Accessed Jan. 14, 2014. <http://www.jointcommission.org/assets/1/6/HAP_NPSG_Chapter_2014 .pdf>.

[10] Cvach M et al. "Clinical Alarms and the Impact on Patient Safety." Initiatives in Safe Patient Care, 2009, pp. 1,5-7.

[11] Graham $\mathrm{KC}$ et al. "Monitor alarm fatigue: standardizing use of physiological monitors and decreasing nuisance alarms." AJCC 2010; 19:28-37.

[12] GE Healthcare. "Critical Care Monitoring Clinical Reference and Troubleshooting Guide." 31 January 2005. Revision A. PM 2007725-001.

[13] Taenzer A et al. "Defining Normality - Postoperative Heart Rate and $\mathrm{SpO}_{2}$ Distribution of In-Hospital Patients." American Society of Anesthesiologists Proceedings 2010:A1466.

[14] Spiess A, Neumeyer N. "An evaluation of R2 as an inadequate measure for nonlinear models in pharmacological and biochemical research: a Monte Carlo approach." $B M C$ Pharmacology 2010, 10:6 